\title{
Behaviour of small-scale turbulence in the turbulent/non-turbulent interface region of developing turbulent jets
}

\author{
By M. Breda and O.R.H. Buxton $\dagger$ \\ Department of Aeronautics, Imperial College London, London, SW7 2AZ, UK
}

(Received Monday 12 ${ }^{\text {th }}$ August, 2019)

Tomographic particle image velocimetry experiments were conducted in the near and intermediate fields of two different types of jet, one fitted with a circular orifice and another fitted with a repeating-fractal-pattern orifice. Breda \& Buxton $(2018 a, b)$ showed that this fractal geometry suppressed the large-scale coherent structures present in the near field and affected the rate of entrainment of background fluid into, and subsequent development of, the fractal jet, relative to the round jet. In light of these findings we now examine the modification of the turbulent/non-turbulent interface (TNTI) and spatial evolution of the small-scale behaviour of these different jets, which are both important factors behind determining the entrainment rate. This evolution is examined in both the streamwise direction and within the TNTI itself where the fluid adapts from a nonturbulent state, initially through the direct action of viscosity and then through nonlinear inertial processes, to the state of the turbulence within the bulk of the flow over a short distance. We show that the suppression of the coherent structures in the fractal jet leads to a less contorted interface, with large-scale excursions of the inner TNTI (that between the jet's azimuthal shear layer and the potential core) being suppressed. Further downstream, the behaviour of the TNTI is shown to be comparable for both jets. The velocity gradients develop into a canonical state with streamwise distance, manifested as the development of the classical tear-drop shaped contours of the statistical distribution of the velocity-gradient-tensor invariants $Q$ and $R$. The velocity gradients also develop spatially through the TNTI from the irrotational boundary to the bulk flow; in particular there is a strong small-scale anisotropy in this region. This strong inhomogeneity of the velocity gradients in the TNTI region has strong consequences for the scaling of the thickness of the TNTI in these spatially developing flows since both the Taylor and Kolmogorov lengthscales are directly computed from the velocity gradients.

\section{Introduction}

Axisymmetric jets have been the subject of numerous studies in the past due to their common use as mixing and thrust producing devices (Ho \& Gutmark 1987). An axisymmetric round jet issuing directly from a contraction produces a flat-topped ("top-hat") velocity profile in the near field and develops towards a turbulent state in the far field by entraining and mixing background (quiescent) fluid into the turbulent stream. Entrainment describes a process through which there is an exchange of momentum, energy, vorticity, mass and other properties between the surrounding fluid and the turbulent stream across the sharp turbulent/non-turbulent interface (TNTI). This process can take 
place in two ways, through engulfment (ingestion of irrotational fluid into the turbulent stream) and nibbling (small-scale diffusion across the interface). These two processes do not exclude each other and based on the state of any coherent structures embedded within the flow one may be more important than the other. Establishing which process is dominant across the evolution of the jet is somewhat open to debate (da Silva et al. 2014), since some authors have suggested that in turbulent jets engulfment is dominant, whilst others have pointed to nibbling. Dahm \& Dimotakis (1987); Dimotakis (2000) suggested that engulfment would be the dominant process in the far field of axisymmetric jets and a similar conclusion was reached by Mungal et al. (1991) for diffusion flames. Contrastingly, Westerweel et al. (2005) pointed to nibbling as the dominant entrainment process in the far field of a jet between $60-100$ jet diameters downstream of the exit.

Examining the state of the TNTI has posed significant challenges in the past due to its very small thickness. The first significant work on this topic (Corrsin \& Kistler 1954) suggested the existence of a viscous laminar superlayer (VSL) which matched the irrotational background to the turbulent, vortical flow. Corrsin \& Kistler (1954) suggested that the thickness of this layer would scale with a viscous lengthscale, usually assumed to be the Kolmogorov lengthscale $\eta$. Note that the outer-most extremity of the TNTI/viscous superlayer is usually referred to as the irrotational boundary. Decadeslater works have suggested that the TNTI is not only composed of the VSL, but also of a turbulent sublayer (TSL) where the vorticity reaches a local maximum and which has a thickness that scales with the Taylor legthscale $\lambda_{f}$ (Bisset et al. 2002; van Reeuwijk \& Holzner 2014) or simply to the radius of the large-scale vortical structures (LVS) near the interface (da Silva \& Taveira 2010).

Traditionally, the smallest scales present in a turbulent flow have been thought to be independent of the large scales, however recent work, particularly at modest Reynolds number, has started to cast doubt on this (Buxton 2015; Fiscaletti et al. 2016a,b). This gives rise to the possibility that as well as directly influencing the engulfment into a turbulent jet the large-scale coherent structures may also indirectly influence entrainment through small-scale nibbling processes, for example if the small scales are "slaved" to the large scales. In this manuscript we examine this by comparing a "classic" round jet with a particular non-circular exit geometry - a repeating fractal pattern. Breda \& Buxton $(2018 a, b)$ recently compared axisymmetric jets fitted with a circular and fractal-shaped orifice with identical open area and at identical Reynolds numbers and showed that the fractal jet substantially suppressed the coherent structures in the near field of the jet in relation to the round jet. In particular, they showed that this modified the entrainment rate into the jet's near field before both jets evolved into an axisymmetric self-similar state further downstream. The objective of this manuscript is thus to try and link this modified entrainment rate to the behaviour of the TNTI and properties of the small-scale turbulence in the near-interface region of these two jets.

The behaviour of the small scales will be examined through the velocity-gradient tensor (VGT), which can be split into a symmetric and skew-symmetric part (or a strain-rate and rotation tensor):

$$
a_{i j}=\frac{\partial u_{i}}{\partial x_{j}}=s_{i j}+\omega_{i j}=\frac{1}{2}\left(\frac{\partial u_{i}}{\partial x_{j}}+\frac{\partial u_{j}}{\partial x_{i}}\right)+\frac{1}{2}\left(\frac{\partial u_{i}}{\partial x_{j}}-\frac{\partial u_{j}}{\partial x_{i}}\right)
$$

Here the flow velocity is split into its mean and fluctuating part following the Reynolds decomposition $U_{i}=\overline{U_{i}}+u_{i}$. Chong et al. (1990); Chacin \& Cantwell (2000) defined the invariants of the VGT, so-called because they remain constant regardless of which coordinate system is used, from the roots of the characteristic equation for $a_{i j}$ written 
as

$$
\xi^{3}+P \xi^{2}+Q \xi+R=0
$$

where $P, Q$ and $R$ are the invariants. The first invariant $P$ describes the negative trace of the $\operatorname{VGT}\left(P=-a_{i i}\right)$ and is therefore zero for an incompressible flow, hence leaving $Q$ and $R$ to describe the flow topology

$$
\begin{aligned}
Q & =\frac{1}{4}\left(\omega_{i} \omega_{i}-2 s_{i j} s_{i j}\right)=Q_{\omega}+Q_{s} \\
R & =-\frac{1}{3}\left(s_{i j} s_{j k} s_{k i}+\frac{3}{4} \omega_{i} s_{i j} \omega_{j}\right)=R_{s}+R_{\omega}
\end{aligned}
$$

where $\omega_{i}$ is the $i^{\text {th }}$ component of the vorticity vector. The invariant $Q$ can be considered as the local excess of swirling over strain rate, with its constituent parts $Q_{\omega}$ being half the magnitude of the enstrophy whilst $Q_{s}=-\varepsilon / 4 \nu$ is proportional to the turbulent kinetic energy dissipation rate $\varepsilon$. Since $P=0, Q$ and $R$ can be used to describe the state of an incompressible fluid element.

The joint probability density function (j.p.d.f.) of $Q$ and $R$ has been found to have a characteristic "tear-drop" shape in a variety of "fully-developed" turbulent flows including homogeneous isotropic turbulence, mixing layers and wall-bounded flows (Soria et al. 1994; Blackburn et al. 1996; Tsinober 2009; Buxton \& Ganapathisubramani 2010). This "ubiquity" led to the belief that the tear-drop shaped j.p.d.f. is a universal signature of "fully-developed" fine-scale turbulent motions (Chacin \& Cantwell 2000; Elsinga \& Marusic 2010). Recently, however, Buxton et al. (2017) has observed this tear-drop shaped distribution of $Q$ and $R$ in the inhomogeneous, spatially-developing near-wake region downstream of a square cylinder suggesting that it may be more ubiquitous than previously thought. Contrastingly, Gomes-Fernandes et al. (2014); Paul et al. (2017) found that the tear-drop shape developed with distance downstream from a fractal-square grid and single-scale grid, respectively. In this manuscript we will also document the spatial development of the $Q-R$ distribution, if it exists, in the round and fractal jets we examine, both in the streamwise direction and in the TNTI-normal direction, i.e. moving from the interface itself into the turbulent bulk. The objective will be to assess what, if any, effect the different state of the coherence in the near fields of the different jets has on the evolution of this $Q-R$ distribution towards a "fully-developed" state.

\section{Methodology}

\subsection{Jet structure}

The flow was generated by an open jet facility at Imperial College London, previously described in Breda \& Buxton $(2018 a, b)$. In order to prevent biasing the particle images with unseeded, quiescent air becoming entrained into the jet a seeded, mild co-flow of air was used as described in Breda \& Buxton $(2018 a, b)$. The exit flow was found to have a sharp "top-hat" mean velocity profile and turbulence intensity $<1 \%$. Two different orifices (round and fractal) of thickness $0.1 \mathrm{~mm}$ and of identical open area $D_{e}^{2}\left(D_{e}=\right.$ $15.78 \mathrm{~mm}$ ) were attached to the nozzle, as shown in fig. 1 . The repeating fractal pattern was formed of three iterations and has a fractal dimension of $D_{f}=1.5$, first described in Nedić et al. (2013). Each jet thus has the same exit flow velocity $U_{e}=9.93 \mathrm{~ms}^{-1}$ for a given volumetric flow rate,

$$
D_{e}=\sqrt{\text { Exit area }} \quad U_{e}=\frac{\text { Volumetric flow rate }}{\text { Exit area }} .
$$




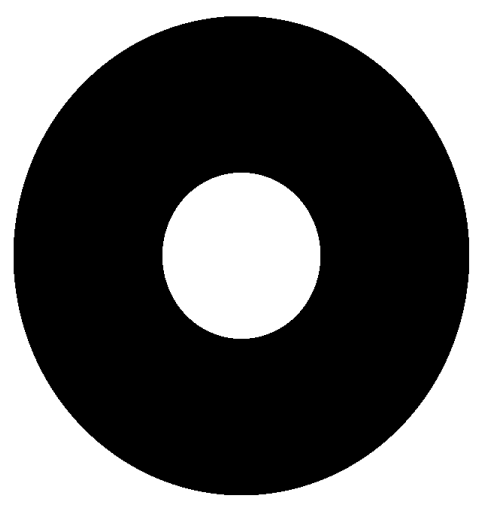

(a)

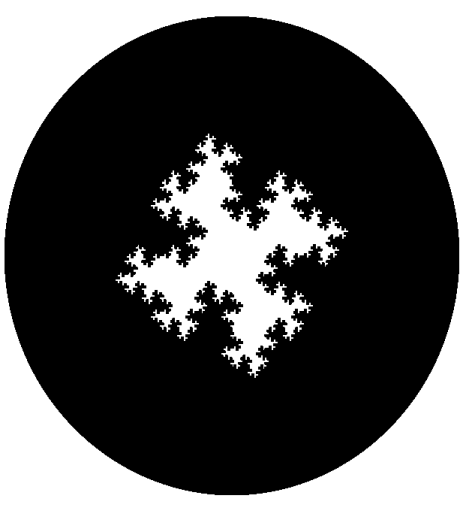

(b)

Figure 1: Exit geometries studied, (a) round and (b) fractal.

meaning that the Reynolds number was kept the same for both jets, defined as

$$
R e_{G}=\frac{U_{e} D_{e}}{\nu}=10^{4}
$$

The flow rates through the jet were carefully controlled using an Omega FMA-26 mass flow controller to ensure that these operating conditions were repeatable.

\subsection{Nomenclature and coordinate system}

The data from the tomographic particle image velocimetry (TPIV) experiments are acquired in a Cartesian coordinate system. This coordinate system is defined with $x$ as the streamwise component and $y$ and $z$ the orthogonal ones. The instantaneous velocities are $U, V$ and $W$ respectively. In some places the Cartesian coordinate system has been converted to a cylindrical one. In that case, $r$ is used to indicate the radial component, whilst $\theta$ indicates the azimuthal one. In the potential core, where the round jet has a "top-hat" velocity profile, the location of the jet centre $(r=0)$ was determined as the minimum value of the root-mean-square (rms) of the streamwise velocity fluctuations. After the azimuthal shear layer coalesces, the location of the jet centre was determined by fitting a Gaussian curve to the streamwise mean velocity profile to find the maximum value. The instantaneous velocity components were then converted to $U_{x}, U_{r}$ and $U_{\theta}$, as shown in fig. 2. The mean (time-average) and fluctuating velocity are subsequently decomposed following the Reynolds decomposition.

\subsection{Tomographic PIV}

The tomographic PIV (TPIV) interrogation volumes were centred on $x / D_{e}=2, x / D_{e}=$ 10 and $x / D_{e}=25$ to study the spatial evolution of the velocity-gradient statistics of the jet, as shown in fig. 3. The three regions were chosen to study the jet in three different phases (Breda \& Buxton 2018b):

- $x / D_{e}=2$ : here the jets are in a state of development. The first Kelvin-Helmholtz vortex rings have already developed and the turbulent kinetic energy is in a production phase, where the velocity fluctuations increase with streamwise distance $x$.

- $x / D_{e}=10$ : the azimuthal shear layer has coalesced and both round and fractal jet have passed the peak of turbulent kinetic energy (along the centre-line). 


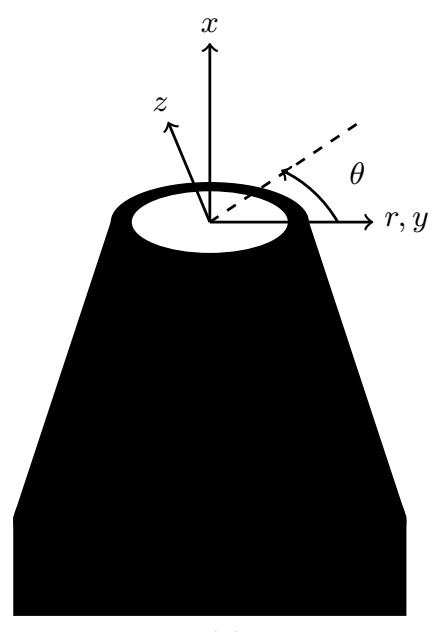

(a)

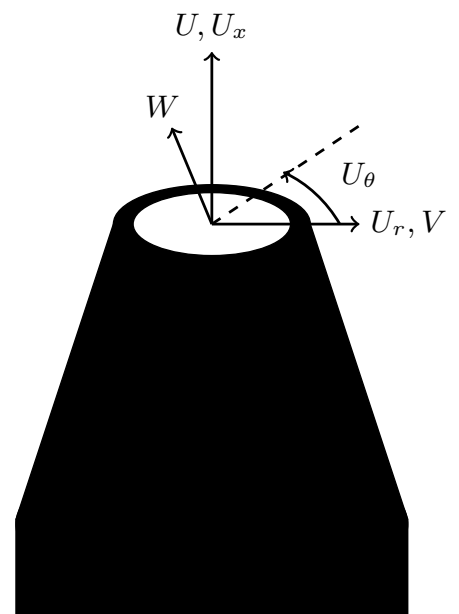

(b)

Figure 2: (a) Jet's coordinate system for the tomographic PIV data and (b) velocity components.

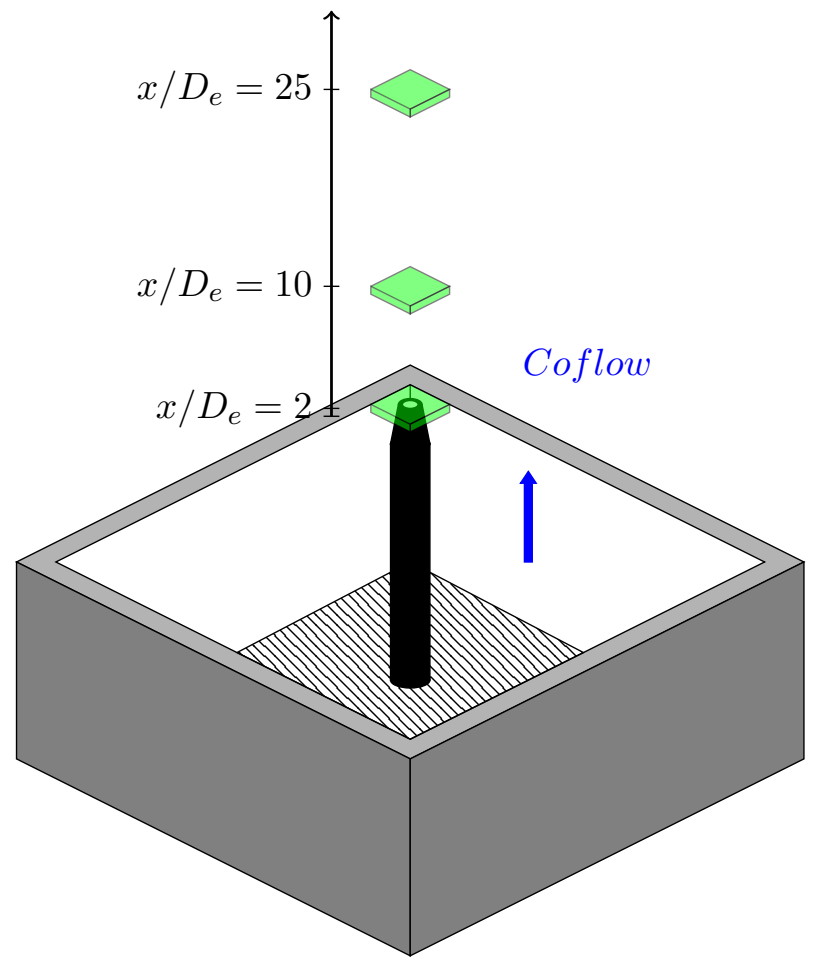

Figure 3: Schematics of volumes studied with TPIV.

- $x / D_{e}=25$ : the mean velocity and Reynolds stresses are self-similar.

Keeping the illuminated volume's thickness to a minimum allows an increased seeding density to be used and hence improves the spatial resolution, which is important to study the velocity gradients. The illuminated-volume thickness was set to be $5 \mathrm{~mm}$ (of which 
$\approx 3 \mathrm{~mm}$ was retained after the PIV processing) with a variable aperture slit in order to compromise between a reasonable spatial resolution and thick enough volume depth whilst ensuring a sharp light intensity gradient at the edge of the volume.

This thickened light sheet was generated by a double cavity Nd:YAG low speed laser running at $15 \mathrm{~Hz}$. The images were acquired with four cameras, 4 Megapixel Imager SX fitted with four lenses of type AF DC-Nikkor $105 \mathrm{~mm} \mathrm{f} / 2 \mathrm{D}$ (f-number f/16). An optical calibration was performed by traversing a dotted plate across the whole volume at the positions $\tilde{x}=0 \mathrm{~mm}, 2.5 \mathrm{~mm} \dagger$ and $5 \mathrm{~mm}$ and acquiring an image from the cameras in each step. The commercial software DaVis was then used to generate the optical three-dimensional calibration knowing the distance between the three streamwise views. In order to improve the calibration quality, a volume self-calibration was completed as discussed by Wieneke (2008). In order to have an homogeneous disparity map, the images were recorded running the co-flow only. After these initial calibrations, images were acquired switching both the co-flow and jet on. The TPIV images were processed using the MART algorithm (6 iterations), a multiplicative algebraic reconstruction (Herman \& Lent 1976).

In order to improve the quality of the reconstruction of the images a dark background, previously acquired, was subtracted. Sliding minimum subtraction, Gaussian smoothing using a $3 \times 3$ pixels kernel to reduce the background noise and sharpening to reduce the number of "ghost particles" were also performed on the raw images, allowing the particle diameter to be of the order of 2 pixels and the ppp (particles per pixel) to be within $0.04-0.05$. In order to reduce the processing time sparse cross-correlation (Discetti \& Astarita 2012) was implemented.

The vectors were reconstructed in three steps: an initial recursive direct correlation‡, followed by two iterations of motion tracking enhancement (MTE) (Novara et al. 2010) to reduce the number of "ghost particles" and a final recursive direct correlation 9 .

Finally, in order to correct the non-zero divergence of the resultant velocity fields, the data were corrected using the divergence correction scheme (DCS) of De Silva et al. (2013). This non-zero divergence is due to experimental noise which, as shown by Buxton et al. (2011), can significantly alter the VGT invariants, obscuring the shape (for example) of the $Q-R$ joint probability density function (j.p.d.f.). The DCS scheme is based on a non-linear optimisation based constraint which minimally alters the acquired velocity field, whilst restricting the magnitude of the divergence to a maximum tolerance. The objective function to be minimised is the ensemble average of twice the "turbulent kinetic energy" added to the measured velocity field, i.e. $\tilde{q}^{2}=\sum_{i=1}^{3}\left\langle\left(\tilde{U}_{i}-U_{i}\right)^{2}\right\rangle$. The divergence error was set to be $\left|\partial \tilde{u}_{i} / \partial x_{i}\right| \leq 10 \mathrm{~s}^{-1} \|$ after a convergence study, which led to an average $\tilde{q} / \bar{U}_{c l}<5 \%$ (compensated by the jet streamwise centreline velocity).

\subsection{Spatial resolution}

The spatial resolution was evaluated based on the measured dissipation rate, $\varepsilon=2 \nu \overline{s_{i j} s_{i j}}$. The Kolmogorov lengthscale was subsequently estimated as $\eta=\left(\nu^{3} / \varepsilon\right)^{1 / 4}$. The transverse

$\dagger$ Here, $\tilde{x}=2.5 \mathrm{~mm}$ is the approximate centre of the laser volume at a given measurement station.

$\ddagger$ The first recursive direct correlation was done in steps of specific correlation volume voxel size (passes): $160 \times 160 \times 160(10) \rightarrow 96 \times 96 \times 96(2) \rightarrow 64 \times 64 \times 64(2)$. The volume overlap was $75 \%$.

I The second recursive direct correlation was: $160 \times 160 \times 160(10) \rightarrow 96 \times 96 \times 96(2)$ $\rightarrow 64 \times 64 \times 64(2) \rightarrow 48 \times 48 \times 48(2)$. The volume overlap was $75 \%$.

$\|$ The notation $~ *$ is used to denote the corrected velocity field for tomographic PIV after the DCS is applied. Later it is dropped since the analysis on the 3D-3C data is based solely on the corrected fields. 
Taylor lengthscale $\lambda_{g}$ was calculated as in Goto \& Vassilicos (2016) by inserting the dissipation rate $\varepsilon$ into

$$
\lambda_{g}=\sqrt{10 \nu \frac{q^{2}}{\varepsilon}},
$$

and this was subsequently converted into the longitudinal $\lambda_{f}$ as

$$
\lambda_{f}=\sqrt{2} \lambda_{g}=\sqrt{20 \nu \frac{q^{2}}{\varepsilon}},
$$

where $q^{2}$ is the turbulent kinetic energy. This led to a spatial resolution (worst case) for the TPIV experiment of $11 \eta$ at $x / D_{e}=2$, of $9 \eta$ at $x / D_{e}=10$ and of $5 \eta$ at $x / D_{e}=25$. These worst case figures are quoted assuming a Kolmogorov length scale computed from the bulk of the turbulent flow, although since the dissipation rate is known to reduce as the irrotational boundary is approached (van Reeuwijk \& Holzner 2014; Buxton et al. 2019) the "local" Kolmogorov length scale is around 20-25\% larger hence the spatial resolution is accordingly finer than these worst-case scenarios. In terms of the longitudinal Taylor lengthscale, the spatial resolution ranged between $0.3 \lambda_{f}$ and $0.1 \lambda_{f}$ at the three locations studied. A summary of the spatial and of the camera's digital resolution $\dagger$ is reported in table 1 .

Figure 4 gives an indication of the spatial resolution and convergence of the dissipation computation achieved in the present study. Comparison is made between the dissipation rate estimated from planar PIV (PPIV) in the study of Breda \& Buxton (2018b) and the present tomographic PIV (TPIV) dataset. Figures 4(c) and (d) show the round and fractal jets probed at the farthest downstream location of $x / D_{e}=25$. The quoted worsecase spatial resolution of Breda \& Buxton (2018b) at this downstream measurement station is $3.7 \eta$, which is sufficient to resolve the velocity gradients (e.g. Worth et al. 2010; Buxton et al. 2011). Since only planar data was available in this case the dissipation rate was estimated by assuming local isotropy from the PPIV data, whereas a direct computation, i.e. $\varepsilon=2 \nu \overline{s_{i j} s_{i j}}$, was used for the TPIV data. It can clearly be seen that the TPIV slightly underestimates the dissipation rate in comparison with the PPIV as may be expected due to the coarser spatial resolution. However, the trends are extremely well captured and at least a portion of the difference between the two estimated dissipation profiles may be down to the assumption of local isotropy. This gives us confidence in our ability to draw meaningful conclusions relating to the VGT phenomenology in the present manuscript. (N.B. the joint p.d.f.s between $Q$ and $R$ extracted from along the irrotational boundary presented in figure 16 gives us further confidence that our spatial resolution is adequate to draw meaningful conclusions.)

Special note should be made here specifically about the spatial resolution at $x / D_{e}=2$ which is given as $11 \eta$ in the worst case scenario. Clearly this is the coarsest of any of the measurement stations (in terms of turbulent length scales) and therefore the velocity gradients are likely to be an underestimate as a result of this spatial filtering. Indeed figures $4(\mathrm{a})$ and (b) show the dissipation rate $\varepsilon$ and $\varepsilon^{-1 / 4}$ which is the constituent part of the Kolmogorov length scale, at the $x / D_{e}=2$ measurement station which is clearly under-resolved. However, in terms of the Taylor length scale the resolution is always better than $0.3 \lambda_{f}$. Buxton (2015) showed that the tear-drop shape of the $Q-R$ distribution relied upon resolving structures of characteristic length scale $>\lambda_{f}$ in addition to the finest scales $\sim \eta$. We finally note that our spatial resolution is thus always finer (including at $\left.x / D_{e}=2\right)$ than the finest filter size $(\Delta=0.4 \lambda)$ used in the large eddy simulation

$\dagger$ The digital resolution refers to how close the camera is to the object, whilst the spatial resolution is also a function of the final interrogation window of the PIV cross-correlation. 


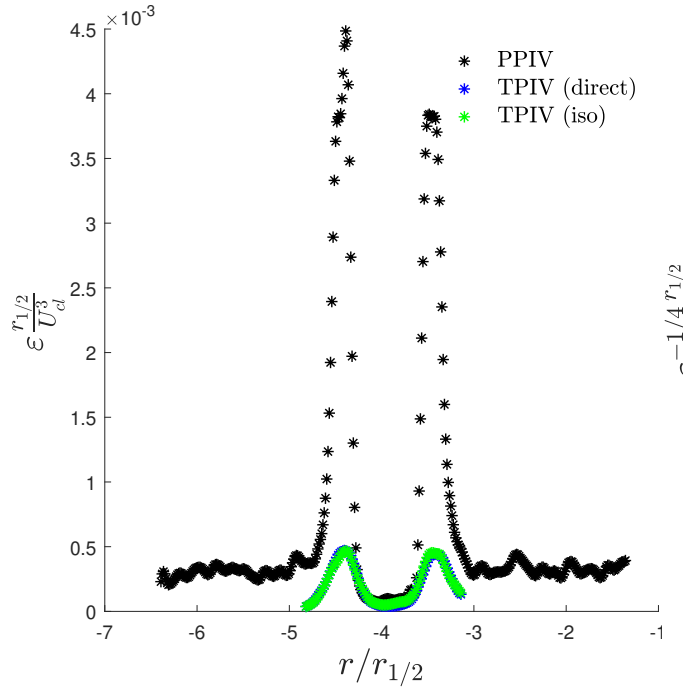

(a)

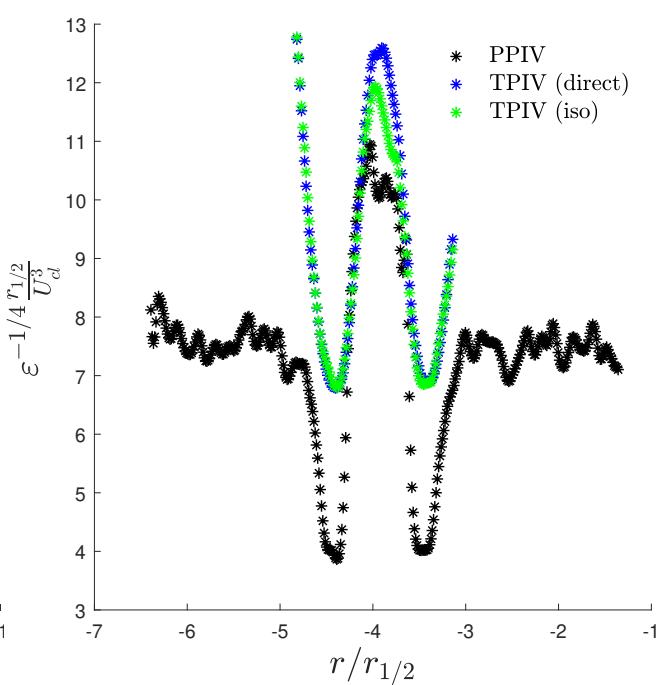

(b)

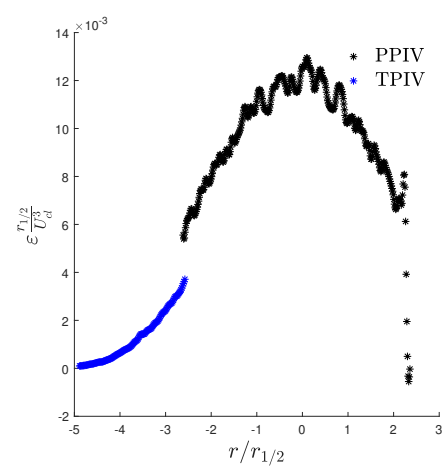

(c)

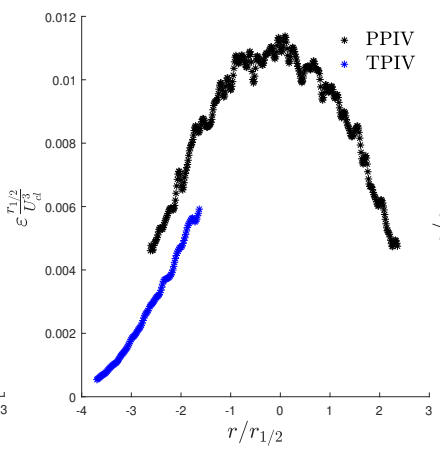

(d)

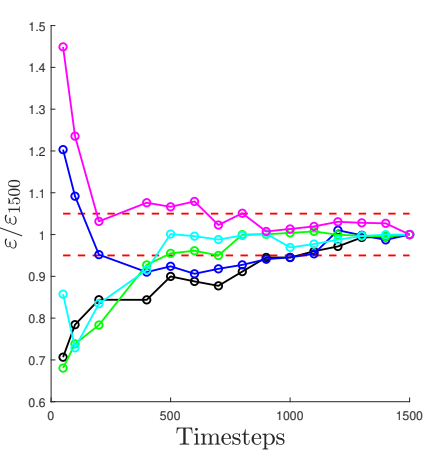

(e)

Figure 4: Comparison of the mean dissipation rate computed from the tomographic PIV (TPIV) of the present study and planar PIV (PPIV) from Breda \& Buxton (2018b) at $x / D_{e}=2(\mathrm{a})$ and (b) and at $x / D_{e}=25(\mathrm{c})-(\mathrm{e})$. Estimates in which the dissipation rate is computed using an assumption of local isotropy from the TPIV data are also presented in (a) and (b) for direct comparison against the PPIV data. (b) presents $\varepsilon^{-1 / 4}$ to illustrate the computation of the Kolmogorov length scale $\eta=\left(\nu^{3} / \varepsilon\right)^{1 / 4}$. (c) and (d) are for the round and fractal jets respectively. (e) Convergence of the calculation for the mean dissipation rate from five data points chosen at random at $x / D_{e}=25$.

study of da Silva (2009) to examine velocity gradient phenomenology in the TNTI region of an axisymmetric jet. For this reason we are confident that we are able to draw at least qualitative conclusions from this measurement station even if we underestimate the VGT phenomenology quantitatively.

Figure 4(e) indicates the convergence of the calculation of the mean dissipation rate. Five data points were chosen at random from the dataset at $x / D_{e}=25$ and the mean dissipation rate was computed as a function of number of snapshots used in the calculation. It can be seen that the results lie well within the $\pm 5 \%$ confidence intervals denoted 
Table 1: Processing details for Planar and Tomographic PIV. The worst case scenario for the spatial resolution is reported.

$\begin{array}{ll}\text { Initial interrogation window } & 160 \times 160 \times 160 \text { voxels } \\ \text { Final interrogation window } & 48 \times 48 \times 48 \text { voxels } \\ \text { Overlap } & 75 \% \\ \text { Spatial resolution ("far field") } & 5 \eta \\ \text { Digital Resolution (px } / \mathrm{mm}) & 45.7 \\ \text { Size of Field of View }(\mathrm{mm}) & 33 \times 29 \times 5\end{array}$

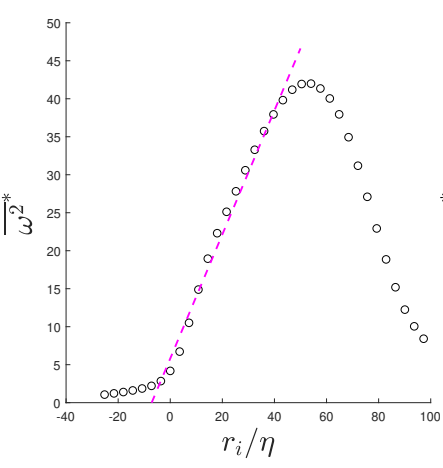

(a) $x / D_{e}=2$

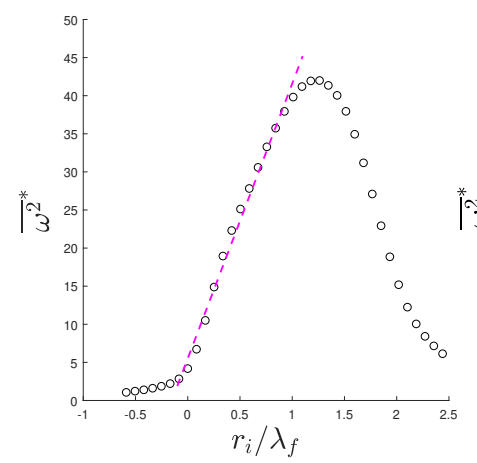

(d) $x / D_{e}=2$

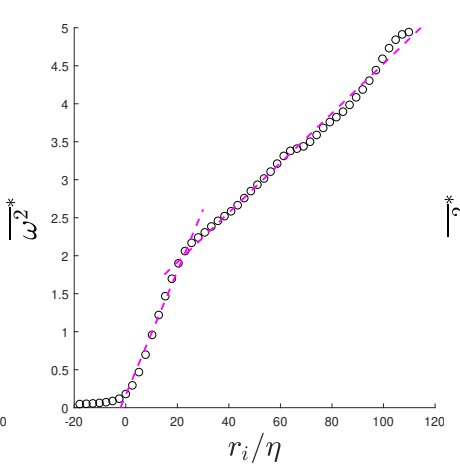

(b) $x / D_{e}=10$

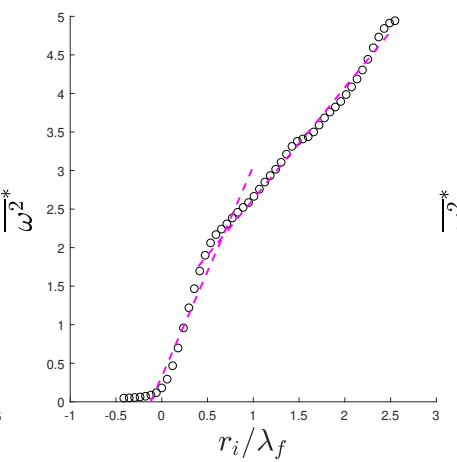

(e) $x / D_{e}=10$

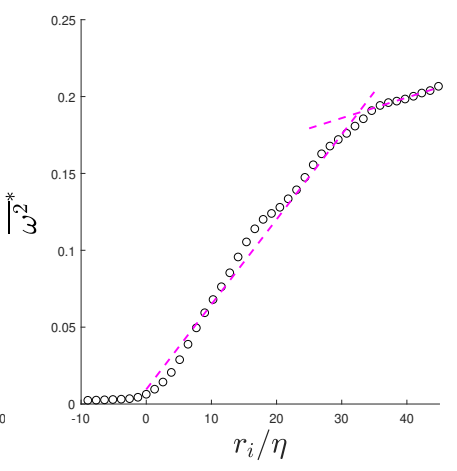

(c) $x / D_{e}=25$

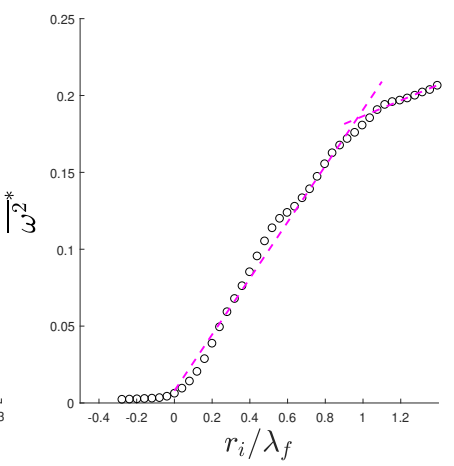

(f) $x / D_{e}=25$

Figure 5: ${\overline{\omega^{2}}}^{*}$ at various streamwise locations for the round jet, compensated by (a,b,c) the mean bulk $\eta$ and (d,e,f) $\lambda_{f}$. The change of growth rate are shown with the dashed magenta lines.

by the dashed lines. We thus estimate the convergence of our dissipation rate estimate to be conservatively $\pm 3 \%$.

\section{Properties of the TNTI}

\subsection{Detection of the TNTI}

The irrotational boundary between the turbulent and non-turbulent fluid (the outermost surface of the TNTI) was identified based on a vorticity-magnitude (twice enstrophy) 
Table 2: Normalised vorticity magnitude thresholds used to define the irrotational boundary.

\begin{tabular}{cccc} 
Jet & $x / D_{e}=2$ & $x / D_{e}=10$ & $x / D_{e}=25$ \\
\hline Round & $\Omega^{*} \geq 1.91$ & $\Omega^{*} \geq 0.40$ & $\Omega^{*} \geq 0.08$ \\
& $\sqrt{\bar{\omega}^{*}} \geq 1.18 \times 10^{-2}$ & $\sqrt{{\overline{\omega^{2}}}^{*}} \geq 1.26 \times 10^{-2}$ & $\sqrt{{\overline{\omega^{2}}}^{*}} \geq 1.95 \times 10^{-2}$ \\
\hline Fractal & $\Omega^{*} \geq 1.91$ & $\Omega^{*} \geq 0.40$ & $\Omega^{*} \geq 0.08$ \\
& $\sqrt{\bar{\omega}^{*}} \geq 1.41 \times 10^{-2}$ & $\sqrt{{\overline{\omega^{2}}}^{*}} \geq 1.41 \times 10^{-2}$ & $\sqrt{{\overline{\omega^{2}}}^{*}} \geq 1.45 \times 10^{-2}$
\end{tabular}

threshold as has been used in previous studies (e.g. Bisset et al. 2002; Holzner \& Lüthi 2011). This threshold is necessarily non-zero due to experimental/numerical noise. Classically, in numerical studies the proportion of fluid identified as being in a turbulent state as a function of chosen vorticity-magnitude threshold yields a plateau region in which the turbulent proportion remains insensitive to small changes (da Silva et al. 2014). This is not possible for our experimental data due to the existence of (unavoidable) experimental noise and the weakly vortical, mild co-flow which was necessary to seed the background in order to avoid biasing the PIV experiments as (potentially unseeded) fluid is entrained into the jet with streamwise distance $x$. As a result the threshold was chosen by use of an iterative procedure in which conditional statistics were produced as a function of distance from the irrotational boundary, $r_{i}$. These statistics were then checked to ensure that they were insensitive to small changes in chosen threshold. These statistics included the conditional mean vorticity-magnitude jumps of figure 5, and ensuring that the joint p.d.f.s between $Q$ and $R$ extracted only from data points along the irrotational boundary contained minimal swirling states (see figure 16).

The threshold (discussed later in the paragraph) of the square root of vorticitymagnitude was selected and the radial distance $r$ from the jet centre was converted into distance $r_{i}$ from the irrotational boundary, where $r_{i}=0$ corresponds to the irrotational boundary and positive $r_{i}$ is in the direction of the turbulent bulk. The conditional mean vorticity-magnitude $\bar{\omega}^{2}$ (i.e. conditioned to the distance from $r_{i}=0$ ) is premultiplied by the kinematic viscosity and dissipation rate

$$
{\overline{\omega^{2}}}^{*}=\overline{\omega^{2}}\left(\frac{\nu}{\varepsilon}\right),
$$

and then plotted in fig. 5a. As shown, the threshold chosen captures the point where ${\overline{\omega^{2}}}^{*}$ sharply increases across the irrotational boundary, whilst for $r_{i}<0$ the vorticity magnitude is approximately zero. The threshold had to be adjusted for the three different streamwise locations studied, since the co-flow would slow down moving up vertically from the jet exit, showing as a consequence a reduced background vorticity. The values chosen were $\Omega^{*}=\left(\overline{\omega^{2}}\right)^{1 / 2} D_{e} / U_{e} \geq 1.91$ for $x / D_{e}=2, \Omega^{*} \geq 0.40$ for $x / D_{e}=10$ and $\Omega^{*} \geq 0.08$ for $x / D_{e}=25$. These thresholds were chosen based on the magnitude of the vorticity but when normalised by the kinematic viscosity and dissipation rate they are observed to be reasonably consistent between measurement stations, in particular for the fractal jet. This information is presented in table 2. A sensitivity study, which is not included for brevity, showed that all subsequent results were insensitive to small changes in threshold. In Bisset et al. (2002), it was shown that for a fully developed turbulent wake $\overline{\omega^{2}}$ 


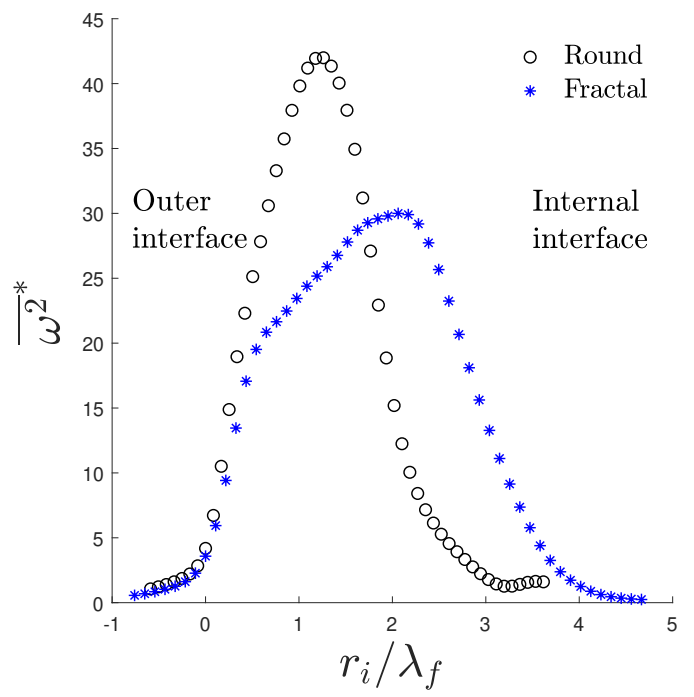

Figure 6: Mean conditional interface for round and fractal jet at $x / D_{e}=2$.

would be close to zero in the non-turbulent zone. It would then sharply increases across the TNTI and finally it would have a different and slower growth rate inside the wake. In figs. 5a to 5c, two different behaviours can be identified as expected. There has been some debate as to the correct scaling of the TNTI thickness in "fully-developed" turbulent flows. Scaling of the TNTI thickness with $\lambda_{f}$ has been widely reported in the literature (e.g. Westerweel et al. 2009; da Silva \& Taveira 2010; Attili et al. 2014) although some doubt has now been cast on this for high Reynolds number flows (e.g. Watanabe et al. 2015; Silva et al. 2018) who suggests that the correct scaling should be $\sim \mathcal{O}(10) \eta$. The purpose of this manuscript is not to examine the scaling of the TNTI thickness for developing flows, we do not compare against a broad range of Reynolds numbers, and so figs. 5a to $5 \mathrm{c}$ depict $r_{i}$ compensated by $\eta$ and figs. $5 \mathrm{~d}$ to $5 \mathrm{f}$ depict $r_{i}$ compensated by $\lambda_{f}$. Without determining a scaling it is merely reported that at $x / D_{e}=2$, the TNTI has a thickness of $\approx 1.2 \lambda_{f}$, at $x / D_{e}=10$ of $\approx 0.7 \lambda_{f}$ and of $\approx \lambda_{f}$ at $x / D_{e}=25$. Moreover, at $x / D_{e}=2$, the TNTI of the fractal jet appears to have a thickness of $\approx 0.6 \lambda_{f}$, which is almost half that of the round jet. This suggests that in the near field, the coherent structures present for the round jet alter the development of the TNTI, increasing the thickness of the outer TNTI, between the jet and the background fluid, whilst the TNTI between the jet and the core appears to have a comparable thickness between the round and fractal jets (fig. 6). Note that at $x / D_{e}=2$ the flow should not be considered as a turbulent flow in the classical sense. Breda \& Buxton (2018a) showed that for the round jet the there was a strong presence of azimuthally coherent vortical structures present at this location that were suppressed to a certain extent in the fractal jet. It is thus more instructive to think of the flow here as being transitional, nevertheless so as not to overburden the reader with additional terminology we refer to the interfacial region adjacent to the internal and external irrotational boundaries as TNTIs. Once the jet's azimuthal shear layer coalesces and the core is absorbed, the TNTI's thickness is initially less than a Taylor lengthscale but then evolves towards $\approx \lambda_{f}$ once the turbulent flow is fully developed in the weakly self-similar region $\left(x / D_{e}=25\right)$. Further remarks are given in section 4.2 . 


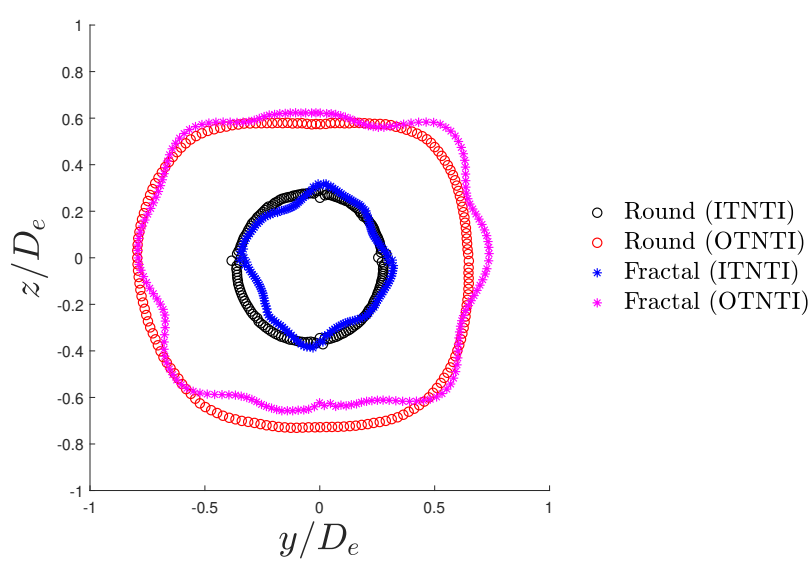

Figure 7: $\overline{\hat{r}}(\theta)$ for round and fractal jet at $x / D_{e}=2$.

\subsection{Tortuosity and radial locus of the TNTI}

Breda \& Buxton $(2018 a, b)$ showed that a consequence of the fractal geometry, considered here, breaking-up the coherent structures in the near field of the fractal jet (in comparison to the round jet) was a reduction of the entrainment and mixing rates. It is hypothesised, therefore, that the different coherent structures may affect the wrinkling of the interfaces and hence the surface area over which diffusion of vorticity may take place. The analysis focuses on two different types of TNTI. At $x / D_{e}=2$, two interfaces are present: the outer interface (OTNTI), between the jet and the background flow, and the inner interface (ITNTI) $\dagger$, between the laminar potential core and the turbulent part of the jet. The tortuosity is calculated for the ITNTI only, since the field of view was not big enough to always capture the complete OTNTI. This was estimated as a function of the averaged contorted interface length $L_{T N T I}$ across all time steps and the average radial distance $\overline{\hat{r}}$ of the TNTI from the jet centre (illustrated in fig. 7):

$$
\text { tortuosity }=\frac{L_{T N T I}}{2 \pi \overline{\hat{r}}} .
$$

The interface length was directly calculated by following the Cartesian coordinates that defined the ITNTI. It is found that the round jet has a tortuosity of $1.49 \pm 0.5 \%$, whilst for the fractal jet it is $1.40 \pm 0.5 \%$. Hence, this hints that the break-up of the coherent structures may (slightly) reduce the wrinkling and the tortuosity of the ITNTI. In order to investigate this further, the probability density function (p.d.f.) of $\hat{r}$ is examined. As shown in fig. 8a, the round jet has wider p.d.f.s at both ITNTI and OTNTI signifying more common large-scale excursions of the TNTIs. However, it is unclear if this is due to the suppression of the large scale structures or simply due to the different exit geometry influencing the mean interface shape.

As shown in fig. 7, both the ITNTI and the OTNTI of the fractal jet have a non-circular shape resembling the exit geometry, hence the radial fluctuations based on a single mean value for $\hat{r}$ may not be actually influenced by the exit geometry. Therefore, in order to evaluate the fluctuations of $\hat{r}$, a different mean $\bar{r}(\theta)$ was calculated every $2^{\circ}$, to account for the different shape of the two TNTIs. The p.d.f. of the fluctuations of $\hat{r}$ is examined in fig. $8 \mathrm{~b}$, where the abscissa has been re-ordered so that when it is positive the TNTI

$\dagger$ Note that at $x / D_{e}=10$ and $x / D_{e}=25$, the terminology TNTI refers to the outer interface of the jet since it is the only one present. 


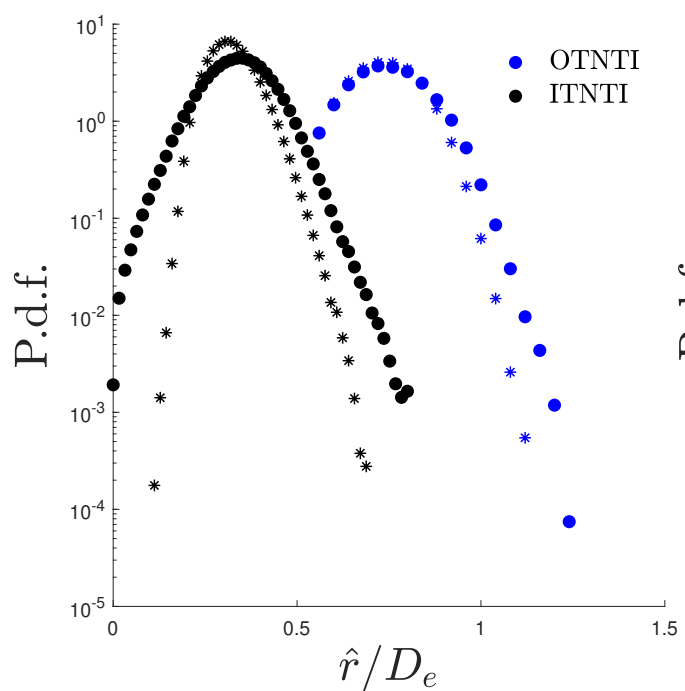

(a)

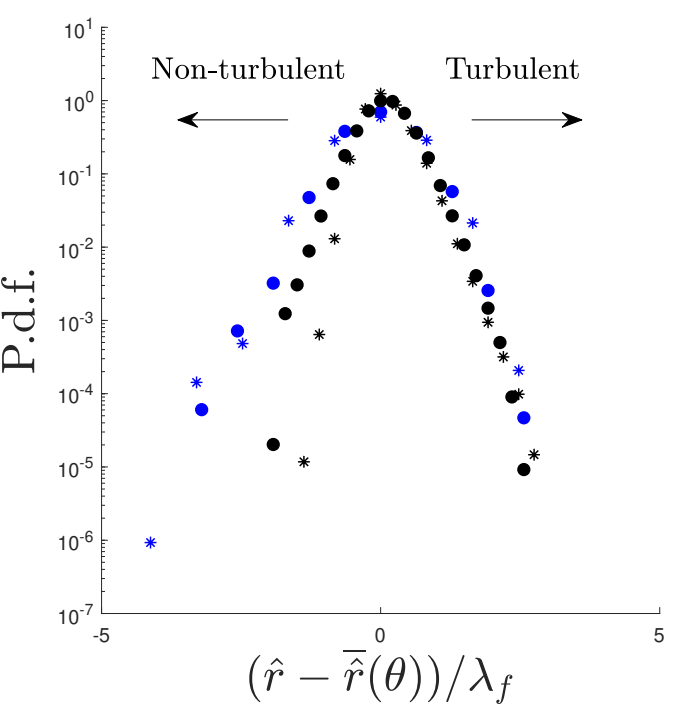

(b)

Figure 8: (a) P.d.f. of $\hat{r}$ for round (o) and fractal (*) jet. (b) P.d.f. of fluctuations of the TNTI based on the angular mean radial distance (same legend applied). Here the abscissa has been oriented so that positive represents the turbulent zone, whilst negative represents non-turbulent areas (either in the jet core or in the quiescent background).

is moving towards the turbulent zone, whilst when negative towards the non-turbulent zone. It is found that the OTNTI and ITNTI of both jets have a comparable positive tail of the p.d.f., suggesting therefore that the movement towards the turbulent zone is not affected by the break-up of the coherent structures. On the negative side of the p.d.f., the OTNTI appears to have a larger tail compared to the ITNTI. Small differences are found on the ITNTI between the two jets, where the round jet has a larger negative tail indicating it is fluctuating more with respect to $\overline{\hat{r}}(\theta)$ due to the influence of the large coherent structures. However, no appreciable differences are found on the negative side of the OTNTI of round and fractal jets, suggesting therefore it is insensitive to the state of the coherent structures. N.B. the mean location of the OTNTI for the round jet is slightly non-circular. We ascribe this to slight asymmetries in the experimental configuration, for example due to a slightly non-uniform co-flow and the small wakes left by the four taut piano wires that were used to keep the central jet structure vertical.

In Breda \& Buxton (2018a), it was suggested that the break-up of the coherent structures produced a thinner shear layer that coalesced further downstream for the fractal jet, influencing the two-point correlations of the velocity fluctuations. This finding is here confirmed in terms of physical thickness, however it is shown that in terms of Taylor lengthscales the shear layer is in effect thicker for the fractal jet (fig. 6). The ITNTI is also more corrugated in the presence of the strong coherent structures of the round jet, but this process is driven by large-scale excursions of the ITNTI - presumably driven by the coherent structures themselves. Interestingly, the primary difference between the two jets are the more common large excursions of the ITNTI into the potential core of the jet, as opposed to the excursions of the OTNTI towards the background fluid. Further downstream, comparable p.d.f.s are found between the two jets for $(\hat{r}-\overline{\hat{r}}(\theta))$ showing that this effect is confined to the near field. Note that the entrainment rates between 


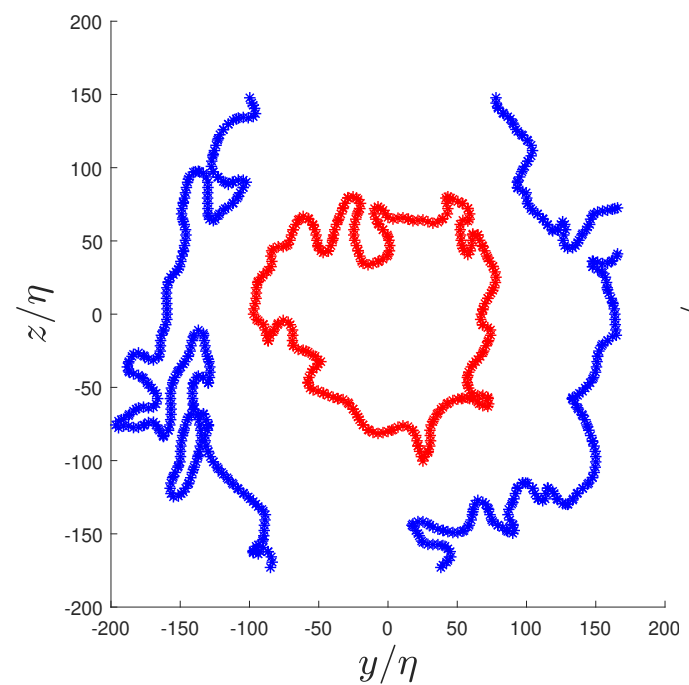

(a) Round jet

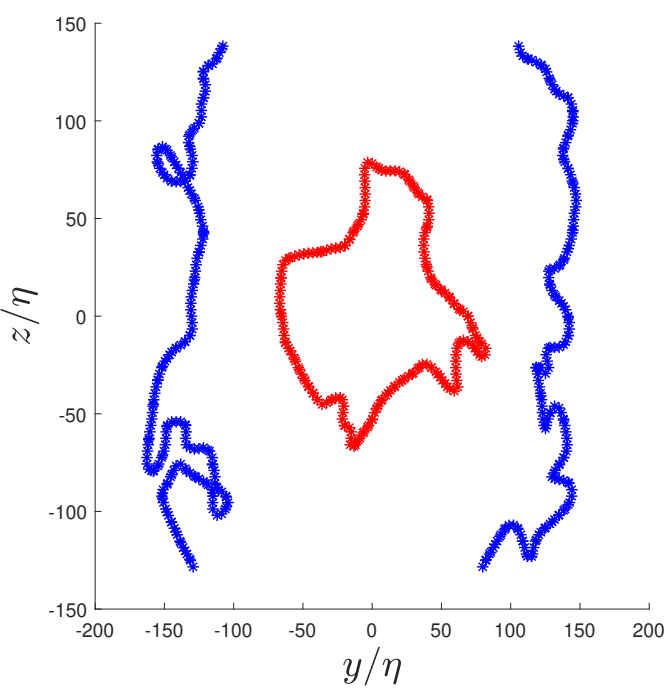

(b) Fractal jet

Figure 9: Instantaneous TNTIs, both inner and outer, with Cartesian spatial coordinates normalised by the Kolmogorov length scale for the round and fractal jets at $x / D_{e}=2$.

the two jets also tended towards the same value further downstream, indicating that the modified entrainment rate in the near field may be a consequence of the modified ITNTI dynamics. Context to this finding is provided in fig. 9a and fig. 9b which show typical instantaneous TNTIs for the round and fractal jets respectively, scaled by the Kolmogorov length scale.

\subsection{Fractal dimension of the TNTI}

Since the work of Sreenivasan \& Meneveau (1986), it has been believed that the the area of a turbulent surface $S$ of a TNTI would follow a power-law in the form $S \sim \Delta^{2-D_{f}}$, where $D_{f}$ is the three-dimensional fractal dimension and $\Delta$ is the side length of a cube. As discussed by Mistry et al. (2018), invoking Reynolds' similarity hypothesis and Kolmogorov scalings leads to a prediction of $D_{f}=2+1 / 3$. Various computational and experimental works have attempted to verify this hypothesis, however strong limitations were posed by the spatial resolution and by the dimensions available $(2 \mathrm{D}$ or $3 \mathrm{D})$. Therefore, rather than evaluating the area of the TNTI, the "fractality" of its length was tested to check it would follow a power law. A popular method to verify this has been the "box counting" technique (Mandelbrot 1982; Sreenivasan et al. 1989; Mistry et al. 2016; Zhou \& Vassilicos 2017). This technique consists in covering the field of view with squares of the same size and counting how many of them contains the TNTI.

Varying the box size $B_{s}$ and counting the number $N$ of boxes containing the TNTI, it should be found that there is a power law in the form $N \sim B_{s}^{m}$. The exponent $m$ is related to the fractal dimension as $D_{f}=1-m$. Therefore, if a Kolmogorov scaling were to be found it would be expected that $m=-4 / 3$. The box-counting technique was applied to the present data at the various streamwise locations to probe the evolution of the TNTI further downstream. In order to test if they follow the Kolmogorov scaling, they are premultiplied so that if a specific scaling was followed, the data would lie on a straight horizontal line.

The data indicates that the TNTI follows a power law at all streamwise locations, how- 


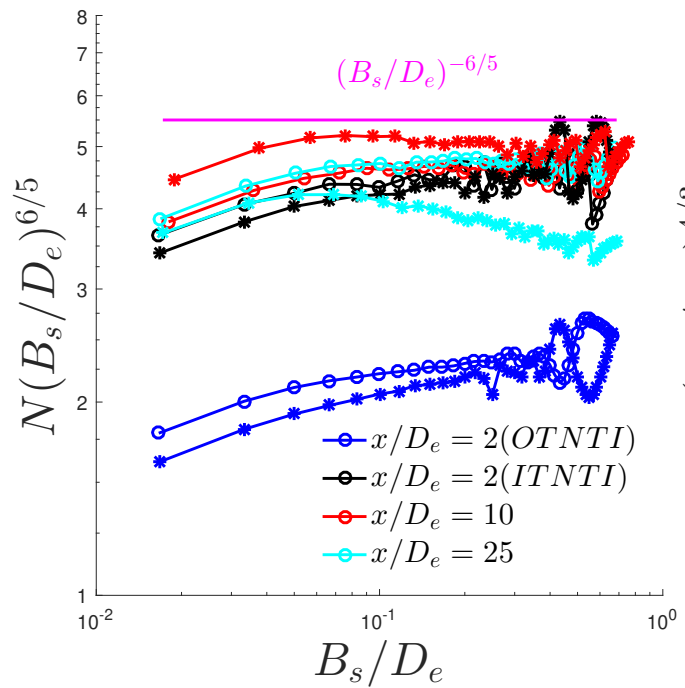

(a) Non-equilibrium scaling

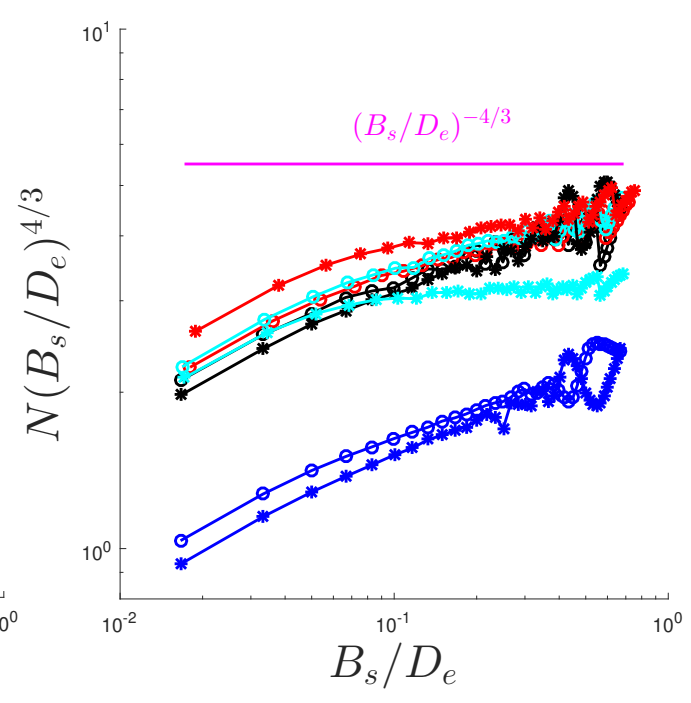

(b) Kolmogorov scaling

Figure 10: Box counting of different interfaces at various streamwise locations for round $(\circ)$ and fractal $(*)$ jets.

ever it does not seem to follow a Kolmogorov scaling. As shown in fig. 10, the interfaces premultiplied by $\left(B_{s} / D_{e}\right)^{6 / 5}$ in fig. 10a appears to follow a $-6 / 5$ scaling rather than the $-4 / 3$ Kolmogorov scaling, as shown in fig. 10b. Only the fractal jet at $x / D_{e}=25$ appears to start approaching the Kolmogorov scaling, whilst the round jet does not. This may be linked to a different state of development of the TNTI at this stage since Breda \& Buxton $(2018 b)$ showed that the number of eddies overturned, computed from integrating the eddy-turnover time and comparing it to the advection time-scale required to advect distance $x$, was substantially different between the round and fractal jets. However, more streamwise locations would need to be probed to confirm this and it should be noted that the p.d.f.s of $\hat{r}$ between the round and fractal jets did not show any discernible differences at this location.

This fractal dimension of $-6 / 5$ has been previously reported as the minimum value measured in jets and plumes by Lane-Serff (1993). The $-6 / 5$ scaling has also been previously discussed by Zhou \& Vassilicos (2017), who completed 3D DNS simulations, looking at the TNTI in 2D planes normal to the mean flow direction of an axisymmetric wake at various streamwise locations. However, in the present study a $-4 / 3$ Kolmogorov scaling was found for the fractal jet at $x / D_{e}=25$ despite non-equilibrium dissipation being measured for this jet at this location, as discussed in Breda \& Buxton (2018b). Therefore, further streamwise locations are needed in order to unveil the transition from a $-6 / 5$ to a Kolmogorov scaling and which phenomena are determining it. The present findings differ from the results of Mistry et al. (2018), who investigated the scaling of the TNTI in the streamwise direction for an axisymmetric jet emitted by a round nozzle. The authors found that up to $x / D_{e}=5$, the TNTI would have a $2 \mathrm{D}$ fractal dimension lower than $4 / 3$ up to $x / D_{e}=5$ and then it would be approximately $4 / 3$ (hence a Kolmogorov scaling) from there up to $x / D_{e}=73(x / D=65$ in the original work where $D$ is simply defined as the round jet diameter), where the jet was self-similar. The authors investigated the jet acquiring data in $r-x$ planes rather than $r-\theta$ planes as in the current work. It may be argued that it is more accurate to count the boxes in a $r-\theta$ plane, as was also done by 
Zhou \& Vassilicos (2017), at a specific streamwise location rather than counting them in the streamwise direction where the scaling may vary due to streamwise inhomogeneity.

\section{Spatial evolution of the microscales of the jet}

As outlined in section 1 one of the objectives of this manuscript is to investigate the spatial variation of the microscale flow physics in the near-interface region. This will reveal the adjustment of the small-scale properties of the turbulence from purely viscousdominated flow physics in the viscous superlayer to the inertially-dominated flow physics in the turbulent bulk portion of the flow. To do this, however, we must first examine the spatial evolution of the velocity gradients in the turbulent bulk of the jets, as the turbulence develops with streamwise distance $x$.

\subsection{State of the microscales of the jet}

\subsubsection{Spatial development of the j.p.d.f. of $Q$ and $R$}

As discussed in section 1 , the smallest scales of the flow are typically thought to be isotropic and statistically homogeneous and in "fully developed" turbulence the joint probability density function (j.p.d.f.) between $Q$ and $R$ is found to have the so-called "tear-drop" shape. However, Buxton et al. (2017) found this shape also in the turbulence production region (the region in which turbulent kinetic energy is an increasing function of $x$ ) for a wake generated by a high-aspect-ratio square cylinder, arguing that the teardrop shaped j.p.d.f. may be a signature of turbulent flows in general. This contrasted previous findings from Gomes-Fernandes et al. (2014) who investigated the evolution of the microscales of grid-generated turbulence at close and intermediate distances from the grid. They found that the j.p.d.f. of $Q$ and $R$ would gradually acquire its tear-drop shape with increasing distance downstream, a finding also observed by Paul et al. (2017). Hence it is now examined to determine whether the tear-drop develops in space or exists from the onset for axisymmetric jets. The region of the flow studied is the bulk of the turbulent flow, where $\Omega^{*}>$ threshold as discussed in section 3.1. Despite the spatial resolution not being sufficient to resolve the Kolmogorov scale in the near field, Buxton (2015) showed that a mix of inertial and dissipative scales is needed to recover the tear-drop shape. Hence, the current spatial resolution is sufficient to study some structural aspects of the small-scale behaviour of the flow.

Figure 11 shows this spatial evolution of the j.p.d.f. of $Q$ and $R$ for both jets. At the farthest upstream station, $x / D_{e}=2$ where the disturbance/randomness has just been introduced, there is a close balance between events of $R>0$ and $R<0$. We acknowledge that the spatial resolution, in terms of Kolmogorov length scale, is coarsest at this location meaning that it is possible that we have been unable to resolve a possible tear-drop shaped j.p.d.f. Nevertheless, this shape closely resembles the shape produced from the synthetic, Gaussian-distributed (i.e. less intermittent than "developed" turbulence) data set produced in Cheng (1996); Chertkov et al. (1999) in which constraints on incompressibility and isotropy have been enforced. Given that we naturally expect the disturbance/randomness to originate at this spatial location the shape of the contours of fig. $11 \mathrm{~b}(\mathrm{a})$ agreeing with a Guassian distribution of VGT states in which incompressibility/isotropy is enforced is somewhat reassuring that indeed these contours are physical rather than a result of lack of spatial resolution. Further, the potential effect of experimental noise at this station was investigated since, as discussed by Buxton et al. (2011), this j.p.d.f. can be highly susceptible to it. A further, more restrictive DCS was applied so that $\left|\partial u_{i} / \partial x_{i}\right| \leq 1 \mathrm{~s}^{-1}$, however the same shaped j.p.d.f. was found, giving additional confidence that this result is physical and not a result of experimental noise. We observe 


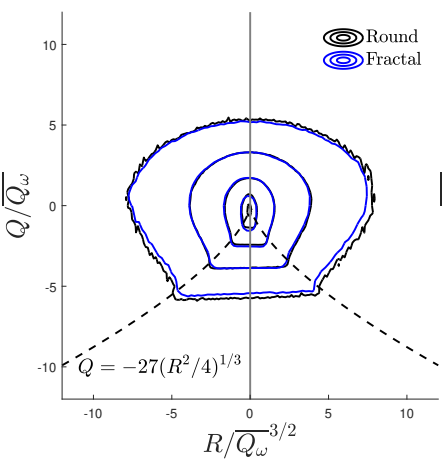

(a) $x / D_{e}=2$

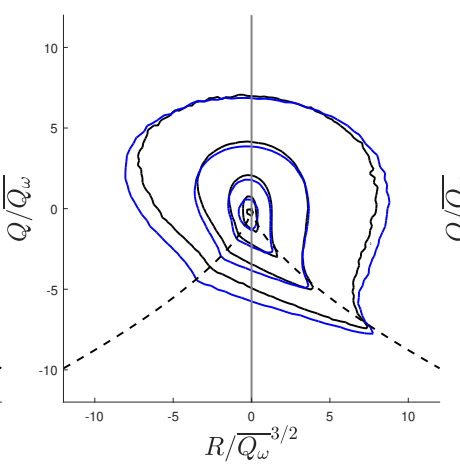

(b) $x / D_{e}=10$

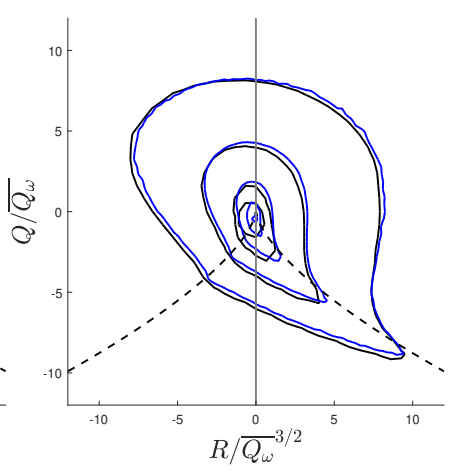

(c) $x / D_{e}=25$

Figure 11: J.p.d.f. of $Q$ and $R$ for the round and fractal jet. Isocontours range from $10^{-4}$ to $10^{0}$. The regions of positive and negative $D$ (the discriminant of the velocity gradient tensor) are separated by a black dashed line.

that the $Q-R$ contours begin to extend along the separatrix $4 Q^{3}+27 R^{2}=0$, which is the discriminant of eq. (1.2), or the so-called Vieillefosse tail (Vieillefosse 1982) at the intermediate station $x / D_{e}=10$. Note that at this location the turbulence intensity along the centre-line of the jet is close to maximum and is decaying (Breda \& Buxton 2018b). At the farthest downstream location, $x / D_{e}=25$, a recognisable tear-drop shape is found which is where the turbulence is the most "fully developed" of any of the measurement stations (Breda \& Buxton 2018b).

In general, when a "classical" tear-drop shape is present, in regions where strain dominates over rotation $(Q<0)$ strain production usually exceeds enstrophy production $(R>0)$, whilst in regions where rotation dominates $(Q>0)$ it is more likely that $R<0$. At $x / D_{e}=2$, the j.p.d.f. is approximately symmetrical and there is no trace of the socalled Viellefosse tail. Moreover, it is found that there is an approximately equal balance between events where strain production exceeds enstrophy production and vice-versa. Therefore, it may be stated that at the start of the turbulence production region of a jet there is a balance between strain production and enstrophy production dominated events. This differs from the grid flow of Gomes-Fernandes et al. (2014), where the separated flow is immediately turbulent. However, similarly to grid-generated turbulence the tear-drop shape clearly evolves to a steady (developed) state with streamwise distance, via transient states originating from a Gaussian-like distribution. This is in contrast to a statistically two-dimensional bluff body wake in which the tear-drop shaped distribution is observed from immediately downstream of the body (Buxton et al. 2017).

\subsubsection{Evolution of enstrophy and strain production}

We now consider some of the constituent terms of the invariant $R$. Equation (1.4) shows that $R$ can be interpreted as a local departure from equilibrium between the inviscid strain-rate and enstrophy amplification terms. The analysis now focuses on the evolution of these terms, $-s_{i j} s_{j k} s_{k i}$ and $\omega_{i} s_{i j} \omega_{j}$ respectively.

In fig. 12a, the strain-rate production/destruction is analysed. The p.d.f.s at the three streamwise locations indicate that at $x / D_{e}=2$ strain destruction is favoured with respect to strain production. However, the flow then develops indicating a prevalence of strain production $\left(-s_{i j} s_{j k} s_{k i}\right)$ further downstream of the jet exit with an amplification of $-s_{i j} s_{j k} s_{k i}$ at the farthest location. The same behaviour was found by Gomes-Fernandes 


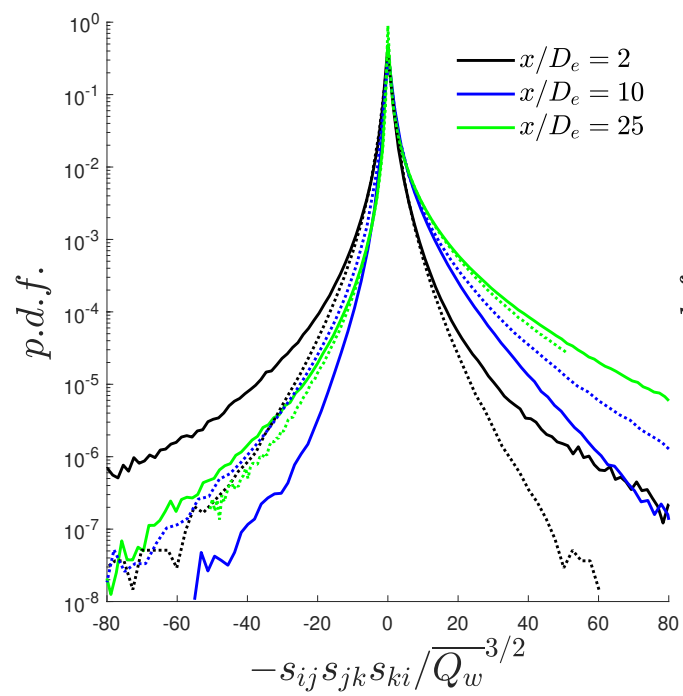

(a)

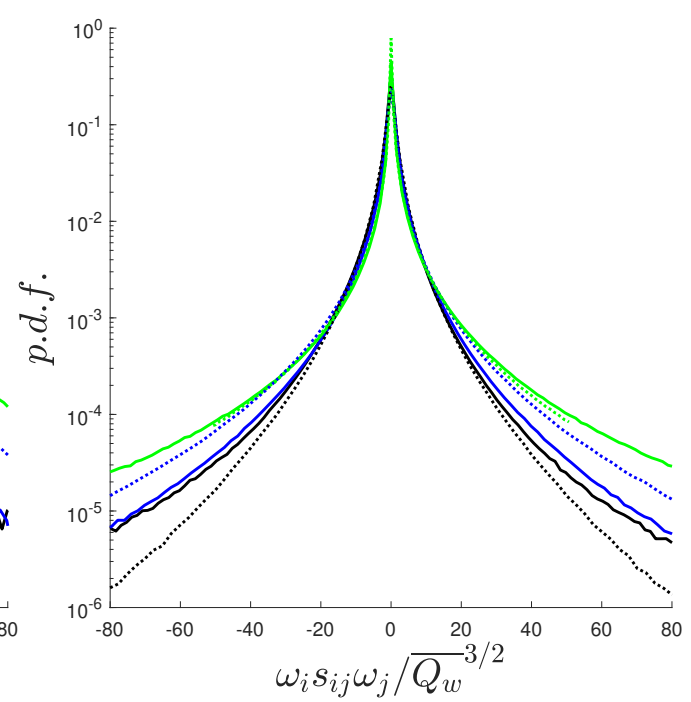

(b)

Figure 12: P.d.f. of enstrohpy and strain for round jet (-) and fractal jet (- -).

et al. (2014), who showed an increasing tendency for positive strain self-amplification $\left(-s_{i j} s_{j k} s_{k i}\right)$ moving further away from their space-filling fractal-square grid. We note that the strain destruction of the round jet appears to reach a minimum level at $x / D_{e} \approx 10$ before regaining its strength further downstream.

As discussed by Taylor (1938), the inviscid vortex stretching term, $\omega_{i} s_{i j} \omega_{j}$, is responsible for allowing the flow's energy to be transferred inertially from the large to the small scales. On average $\left\langle\omega_{i} s_{i j} \omega_{j}\right\rangle>0$, indicating therefore that vortex stretching is favoured over compression, and this has been verified in a wide range of flows (Tsinober et al. (1992) amongst others). At $x / D_{e}=10$ and $x / D_{e}=25$, the ensemble average of $\omega_{i} s_{i j} \omega_{j}$ is positive for both jets, in accordance with previous literature. This is also shown in fig. $12 \mathrm{~b}$, where the p.d.f. indicates a slight skewness towards the positive side. However, at $x / D_{e}=2\left\langle\omega_{i} s_{i j} \omega_{j}\right\rangle$ is negative for both jets, indicating a predominance of vortex compression over stretching.

\subsubsection{Strain-vorticity coupling}

As discussed by Betchov (1956), the vortex stretching term can be rewritten as $\omega_{i} s_{i j} \omega_{j}=$ $\omega^{2} s_{i}\left(\boldsymbol{e}_{\boldsymbol{i}} \cdot \boldsymbol{\omega}\right)^{2}$, where $s_{i}$ are the eigenvalues of the strain-rate tensor, $\boldsymbol{e}_{\boldsymbol{i}}$ are the corresponding (unit) eigenvectors, $\boldsymbol{\omega}$ a unit vector indicating the direction of the vorticity vector and summation over index $i$ is implicit. Here, $s_{3}$ represents the compressive eigenvalue (always negative), $s_{1}$ the extensive one (always positive) and $s_{2}$ the intermediate one (either positive or negative). These eigenvalues can be ordered as $s_{1} \geq s_{2} \geq s_{3}$ and for an incompressible flow $\sum_{i=1}^{3} s_{i}=0$.

As shown in fig. $13 \mathrm{a}$, the symmetrical p.d.f. of $\omega_{i} s_{i j} \omega_{j}$ is confirmed at $x / D_{e}=2$. At $x / D_{e}=10, \omega^{2} s_{1} \cos ^{2}\left(\boldsymbol{e}_{\mathbf{1}}, \boldsymbol{\omega}\right)$ and $\omega^{2} s_{3} \cos ^{2}\left(\boldsymbol{e}_{\mathbf{3}}, \boldsymbol{\omega}\right)$ have an almost symmetrical p.d.f., whilst $\omega^{2} s_{2} \cos ^{2}\left(\boldsymbol{e}_{\mathbf{2}}, \boldsymbol{\omega}\right)$ has a clear skewness for vortex stretching which persists up to $x / D_{e}=25$. At this station, the p.d.f.s of $\omega^{2} s_{1} \cos ^{2}\left(\boldsymbol{e}_{\mathbf{1}}, \boldsymbol{\omega}\right)$ and $\omega^{2} s_{3} \cos ^{2}\left(\boldsymbol{e}_{\mathbf{3}}, \boldsymbol{\omega}\right)$ are again similar, even though $\omega^{2} s_{3} \cos ^{2}\left(\boldsymbol{e}_{\mathbf{3}}, \boldsymbol{\omega}\right)$ appears to be slightly more intermittent, whilst the clear skewness of $\omega^{2} s_{2} \cos ^{2}\left(\boldsymbol{e}_{\mathbf{2}}, \boldsymbol{\omega}\right)$ points to the importance of the inter- 


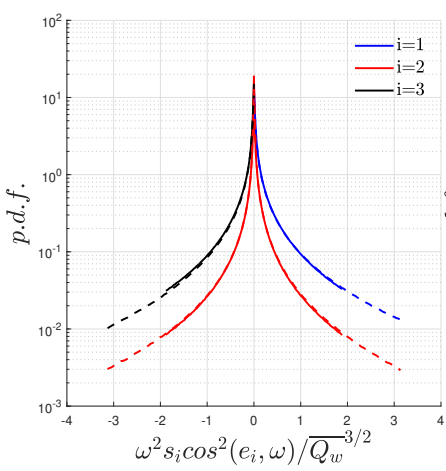

(a) $x / D_{e}=2$

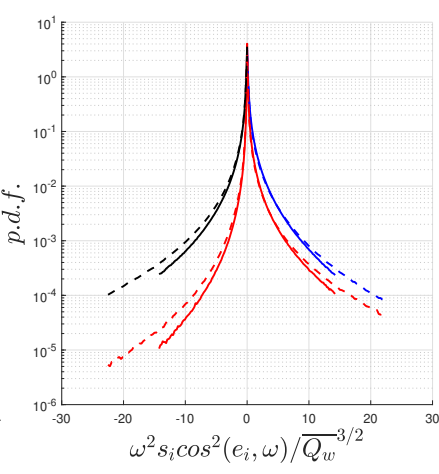

(b) $x / D_{e}=10$

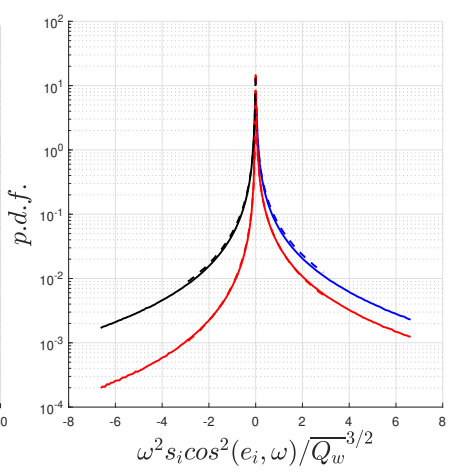

(c) $x / D_{e}=25$

Figure 13: P.d.f. of the spatial evolution of $\omega^{2} s_{i} \cos ^{2}\left(\boldsymbol{e}_{\boldsymbol{i}}, \boldsymbol{\omega}\right)$ for round jet (-) and fractal jet $(--)$.

mediate eigenvalue/vector $s_{2} / \boldsymbol{e}_{2}$ in the process of vortex stretching. The findings in fig. 13 show some similarities to the evolution of vortex stretching studied by GomesFernandes et al. (2014). The authors found that, up to the turbulence production peak, $\omega^{2} s_{i} \cos ^{2}\left(\boldsymbol{e}_{\boldsymbol{i}}, \boldsymbol{\omega}\right)$ displayed symmetrical p.d.f.s, whilst in the decay region $\omega^{2} s_{2} \cos ^{2}\left(\boldsymbol{e}_{\mathbf{2}}, \boldsymbol{\omega}\right)$ would show a clear skewness towards positive values. However, differently from the present study, the authors found that $\omega^{2} s_{1} \cos ^{2}\left(\boldsymbol{e}_{\mathbf{1}}, \boldsymbol{\omega}\right)$ would have a wider p.d.f. compared to $\omega^{2} s_{3} \cos ^{2}\left(\boldsymbol{e}_{\mathbf{3}}, \boldsymbol{\omega}\right)$, suggesting the behaviour of $\omega^{2} s_{i} \cos ^{2}\left(\boldsymbol{e}_{\boldsymbol{i}}, \boldsymbol{\omega}\right)$ might be intimately related to the particular flow studied.

We shall now attempt an explanation for the non-canonical behaviour of the velocity gradients at $x / D_{e}=2$ and why this canonical behaviour evolves towards $x / D_{e}=25$. Clearly the coherent structures wane in influence with streamwise distance which is coupled to a departure from Gaussianity of the velocity gradients, which is necessary to enforce non-linear behaviour such as $\left\langle\omega_{i} s_{i j} \omega_{j}\right\rangle>0$. In addition, near the jet exit, the evolution of the strain field is somewhat constrained by the presence of the potential core, where the ITNTI and the OTNTI act like barriers to the development of the vortical structures. This idea is explored further in section 4.3. We may define a characteristic lengthscale separating the OTNTI and ITNTI as $\mathcal{L}_{\omega}=\hat{r}_{O T N T I}-\hat{r}_{I T N T I}$, or simply the difference between the mean radius of the OTNTI and of the ITNTI. It is hypothesised that $\mathcal{L}_{\omega}$ may constrain the evolution of the turbulent structures if there is insufficient space to achieve a natural scale separation. Ishihara et al. (2013) noted in their DNS work on homogeneous turbulence that thin layers of thickness $2.8 \lambda_{f}\left(4 \lambda_{g}\right)$ exist, which consist of clusters of strong vortex tubes that contribute significantly to the mean dissipation rate. Elsinga et al. (2017) confirmed these findings for homogeneous turbulence (DNS) suggesting that the non-local strain width was $2.8 \lambda_{f}\left(4 \lambda_{g}\right)$ and that the local strain scaled with $\eta$. They were then able to attribute Reynolds number transitions in turbulence behaviour to those Reynolds numbers at which $\lambda_{f} / \eta$ was sufficiently large to allow such structures to form. In the present study, it was found that $\overline{\mathcal{L}_{\omega}} \approx 1.8 \lambda_{f}$ for the round jet and $\overline{\mathcal{L}_{\omega}} \approx 2.4 \lambda_{f}$ for the fractal one.

Since $\overline{\mathcal{L}_{\omega}}$ is less than $2.8 \lambda_{f}$ for both jets, this may suggest that the physical space available is not sufficient for the non-local strain to develop. Hamlington et al. (2008) showed that strain-rate and vorticity are linked by a non-linear, non-local coupling. They showed that the non-local strain-rate contributed significantly to the vortex stretching since the vorticity vector preferentially aligned with the background extensional strain-rate, i.e. $\tilde{e}_{1}$, 


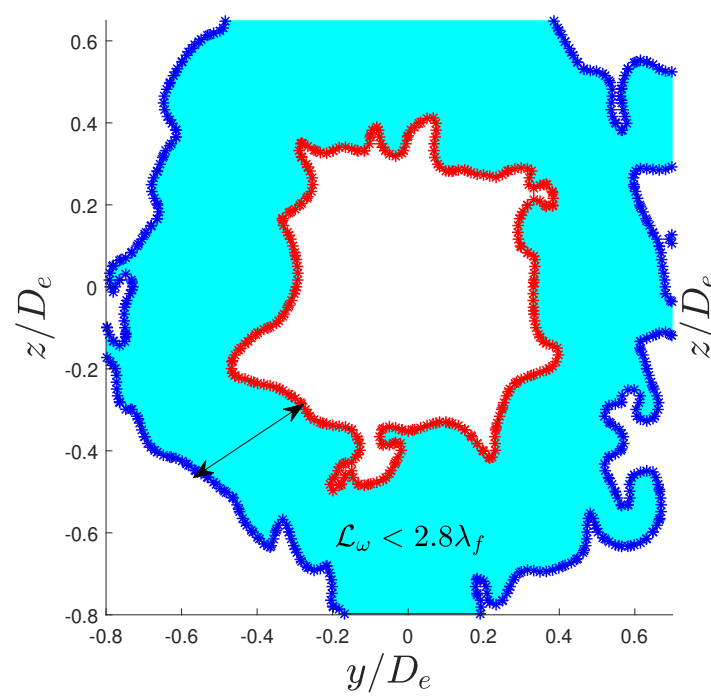

(a) $x / D_{e}=2$

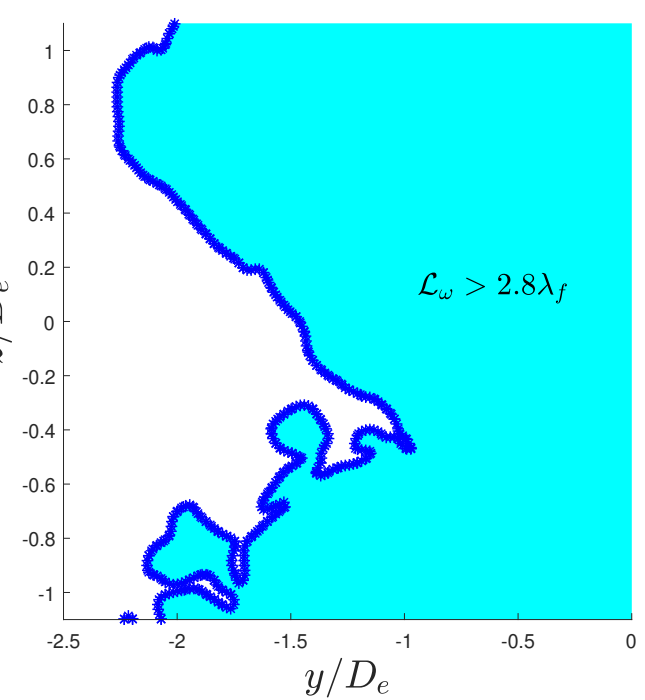

(b) $x / D_{e}=10$

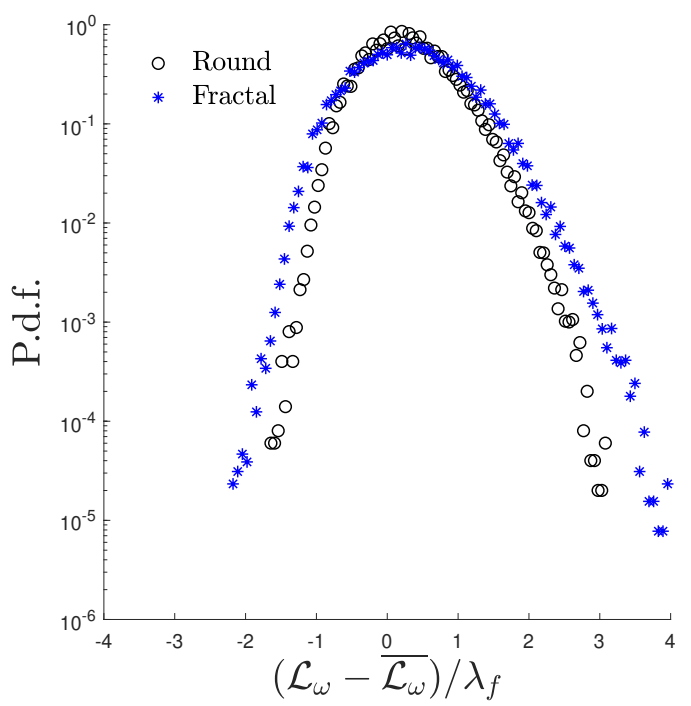

(c) $x / D_{e}=2$

Figure 14: Example of turbulent lengthscales (a) constrained between the OTNTI and the ITNTI and (b) unconstrained (Round jet). The turbulent area is shaded in light blue. (c) P.d.f. of the fluctuations of $\mathcal{L}_{\omega}$ with respect to its mean $\overline{\mathcal{L}_{\omega}}$.

with its correspondingly high magnitude eigenvalue $\tilde{s}_{1}$, whereas it preferentially aligned with the intermediate local strain-rate $\boldsymbol{e}_{\mathbf{2}}$ which has the lowest magnitude eigenvalue of any of the (local) principal strain-rates. We thus postulate that the blocking effect of both TNTIs hinders vortex stretching through inhibiting the contribution of non-local strain. Later on, when the shear layer coalesces and the ITNTI is absorbed into the turbulent bulk, the strain has all the physical space necessary to evolve towards its (natural) non-local state. A schematic is shown in figs. $14 \mathrm{a}$ and $14 \mathrm{~b}$.

The round and fractal jets have a comparable p.d.f. of the fluctuations of $\overline{\mathcal{L}_{\omega}}$, where 


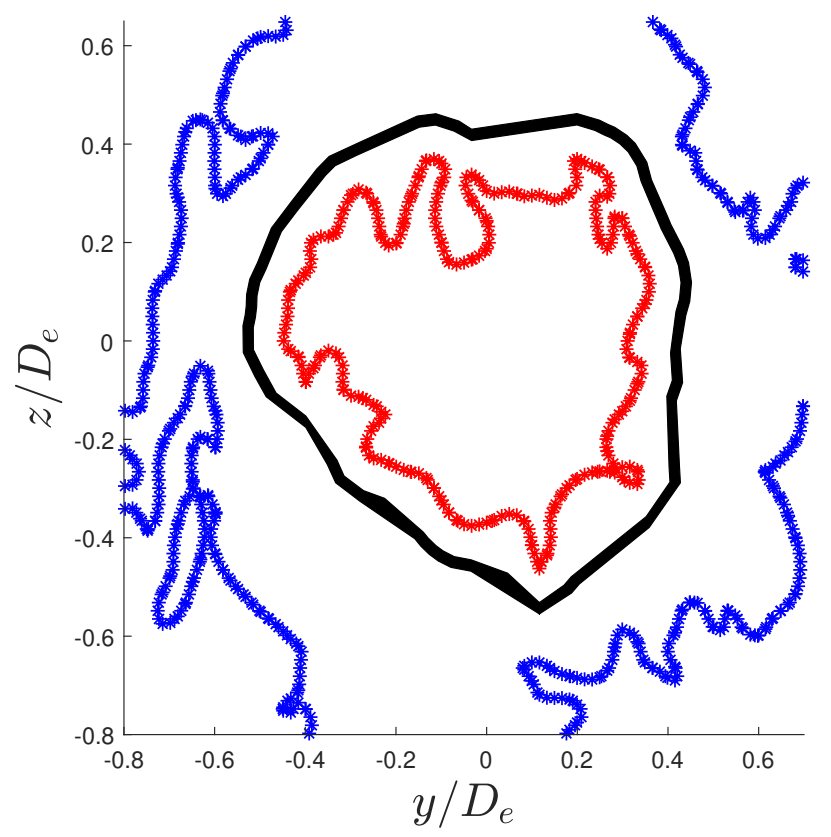

Figure 15: Example of an area probed between $15-20 \eta$ at $x / D_{e}=2$ (black). The ITNTI is shown in red and the OTNTI in blue (Round jet).

the positive side of the p.d.f. is wider than the negative one. In general, the p.d.f. of the fractal jet is only marginally wider than the one of the round jet as shown in fig. 14c, suggesting that the break-up of the coherent structures does not significantly alter the behaviour of $\mathcal{L}_{\omega}$.

\subsection{Small-scale evolution across the TNTI}

We now focus on the spatial evolution of the state of the velocity gradients across the TNTI. This is done by extracting the joint p.d.f. between $Q$ and $R$ from specific spatial regions at a given distance from the irrotational boundary. The intervals chosen were $0-5 \eta, 5-10 \eta, 10-15 \eta$ and so on up to $55-60 \eta$. An example is shown in fig. 15 . It is noted that due to the low spatial resolution the findings in the near field of the jet $\left(x / D_{e}=2\right)$ may appear filtered with respect to the stations further donwstream.

The data presented in fig. 16 suggests that at $r_{i} \approx \lambda_{f}$ the contours become comparable between the evolving TNTI and the turbulent bulk region. For an incompressible flow $\left(P=-a_{i i}=0\right)$ the discriminant of eq. (1.2) is given by $D=27 / 4 R^{2}+Q^{3}$ and hence the curve $D=0$, marked onto the figures, separates purely real roots for $D<0$ (straining motion only) from one real and a complex conjugate pair of roots for $D>0$ (swirling motion). Examining in detail the contours at the interface location, it is found that at $x / D_{e}=10$ and $x / D_{e}=25$ all the points are associated with $D<0$, indicating purely straining motion. This is consistent with the original intuition of Corrsin \& Kistler (1954) and the DNS results of da Silva \& Pereira (2008), who found the same behaviour at the irrotational boundary of a plane jet and provides further validation of our irrotational boundary detection threshold. The fact that this is not observed at $x / D_{e}=2$ is likely a result of the spatial filtering introduced as a consequence of the coarser spatial resolution (in terms of $\eta$ ) at this measurement station coupled to the stronger background freestream turbulence (c.f. the increased $\Omega^{*}$ threshold at $x / D_{e}=2$ in table 2) resulting from the 


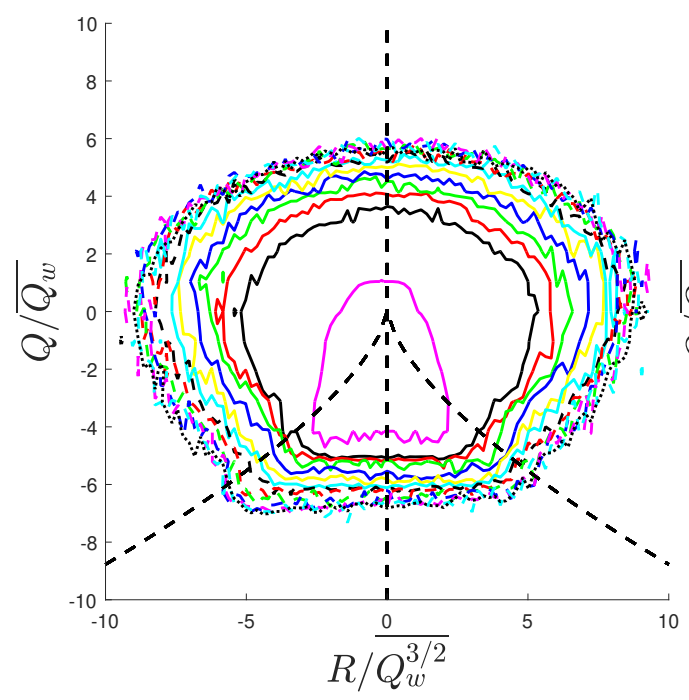

(a) $x / D_{e}=2(\mathrm{OTNTI})$

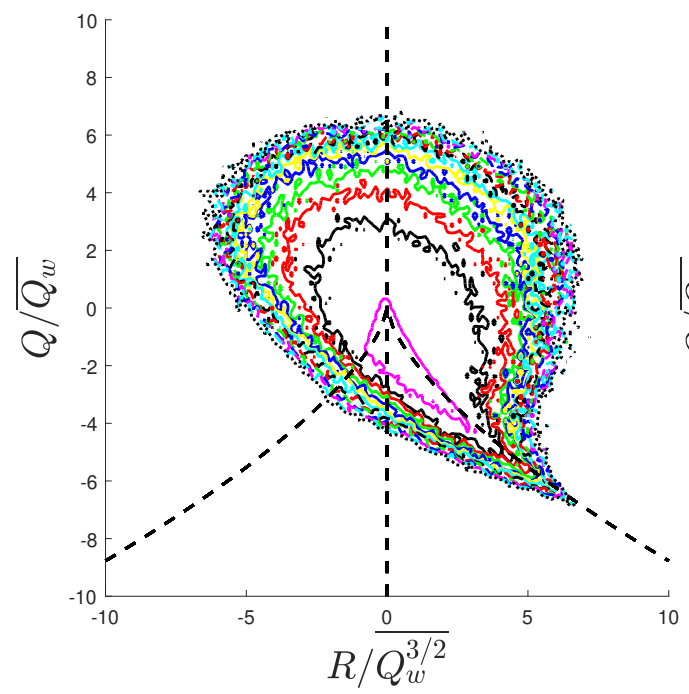

(c) $x / D_{e}=10$

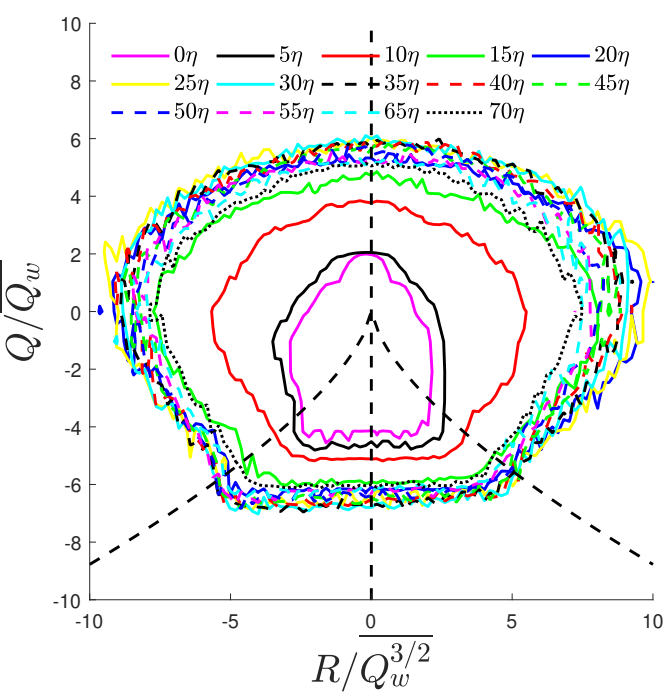

(b) $x / D_{e}=2($ ITNTI)

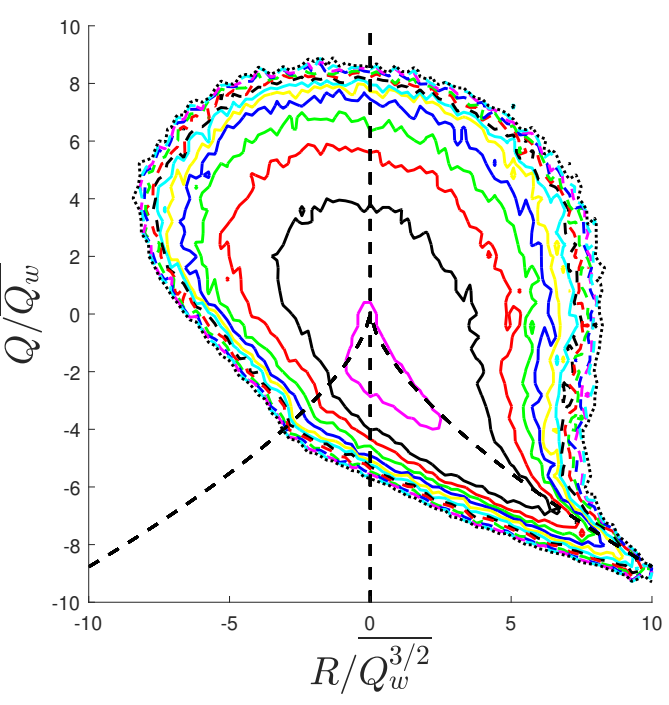

(d) $x / D_{e}=25$

Figure 16: J.p.d.f. of $Q$ and $R$, at a isocontour of $10^{-2}$ for the round jet (a comparable behaviour is found for the fractal jet as well).

mild co-flow. The contribution of swirling dynamics, typically associated with vortex stretching/compression (Buxton \& Ganapathisubramani 2010), increases with $r_{i}$, i.e. within the turbulent sublayer of the TNTI. Note that the tear-drop shape begins to develop over a distance as small as $5 \eta$ at $x / D_{e}=10$ and $x / D_{e}=25$. This is similar to the finding of Watanabe et al. (2017) who showed that the tear-drop shape emerged after $\approx 5.5 \eta$ in their DNS of shear-free turbulence.

These findings suggest that the microscale properties vary inside the turbulent sublayer, whose thickness is comparable to $\lambda_{f}$. After that, the microscale properties remain the same as in the turbulent bulk at each streamwise location. Given that the present 
study considers two different jets, with differing contributions from large-scale coherent motions, at multiple streamwise measurement stations along the jets' streamwise development this is a robust finding.

\subsection{Consequences for the scaling of the TNTI thickness}

Whilst the thickness of the TNTI has been reported to scale with $\lambda_{f}$ (e.g. Bisset et al. 2002; Westerweel et al. 2005; Attili et al. 2014) or $\eta$ (Silva et al. 2018) this literature has not covered from which regions of the turbulent fluid these two lengthscales should be computed. Often, an average value of these two lengthscales has been calculated in the turbulent bulk or along the centre-line of the flow. This is physically meaningful for a sufficiently "fully-developed" flow that the profiles of the relevant turbulent statistics have become self-similar. In such a flow the profile of the mean dissipation rate, a constituent in the computation of both $\lambda_{f}$ and $\eta$ can be correctly scaled with just a single value of the dissipation, typically the centre-line value $\varepsilon_{0}$. In developing, i.e. non-self-similar, flows such as the jets studied here this is not the case and it is not clear from where the mean dissipation rate should be extracted within the flow. It is usual to extract the value of $\lambda_{f}$ or $\eta$ from within the turbulent bulk under the assumption that the turbulent behaviour of the TNTI is "slaved" to the turbulent dynamics of the bulk flow (e.g. Attili et al. 2014).

However, for shearless interfaces at least, doubt has been cast on this assumption. For example da Silva (2010) argued that for shearless flows the TNTI consisted simply of the dissipative intense vorticity structures immediately adjacent to the irrotational boundary. More recently, Watanabe et al. (2017) made reasonable predictions of the conditional statistics across TNTIs using a model of a Burgers vortex placed within the turbulent sublayer of the TNTI of shear-free turbulence. In both cases the properties of the TNTI itself was shown to depend on the turbulence characteristics of the TNTI layer as well as the turbulent bulk and hence it is reasonable to probe the variability of turbulent length scales in this region. Figure 16 clearly shows that the state of the velocity gradients, and hence dissipation rate, is highly variable across the TNTI region. This opens the question as to whether a turbulent lengthscale computed from the turbulent bulk is relevant to a local scale computed within the TNTI region.

Therefore, the evolution of $\eta$ and $\lambda_{f}$ is examined "locally", to verify how they change across the TNTI. To do so, the two local scales $\hat{\lambda}_{f}$ and $\hat{\eta}$ are introduced. These are defined as $\hat{\eta}=\left(\nu^{3} / \hat{\varepsilon}\right)^{1 / 4}$ and $\hat{\lambda}_{f}=\left(20 \nu \hat{q}^{2} / \hat{\varepsilon}\right)^{1 / 2}$ where $\hat{\varepsilon}$ is a local dissipation rate and $\hat{q}^{2}$ is a local turbulent kinetic energy computed from an ensemble situated at a particular distance from the TNTI. The local scales are computed at the irrotational boundary first and then in "slices" of thickness $5 \eta$ at various distances away from the TNTI in the same intervals as before: $0 \eta, 0-5 \eta, 5-10 \eta, 10-15 \eta$ and so on. Only the data points which are above the vorticity threshold previously discussed are included in the calculation. The findings are also evaluated in terms of the mean ratio between bulk $\lambda_{f}$ and $\eta$ to verify if the local quantities vary across the TNTI only or not. The ratio for the two jets at various streamwise distances is shown in fig. 17. This ratio represents scale separation in the flow and so the fact that both jets have an identical global Reynolds number of $\operatorname{Re}_{G}=10^{4}$ but different $\lambda_{f} / \eta$ ratios illustrates the effects that the fractal geometry has on the inertial/dissipative scales of the turbulence. Note that the difference reduces with streamwise distance as the turbulence develops which is consistent with our other findings.

As shown in fig. 18, both $\hat{\lambda}_{f}$ and $\hat{\eta}$ vary significantly from the irrotational boundary towards the turbulent bulk. The greatest ratio between both lengthscales and their mean bulk value is at the interface. At the three streamwise locations, $\hat{\lambda}_{f}$ appears to evolve up 


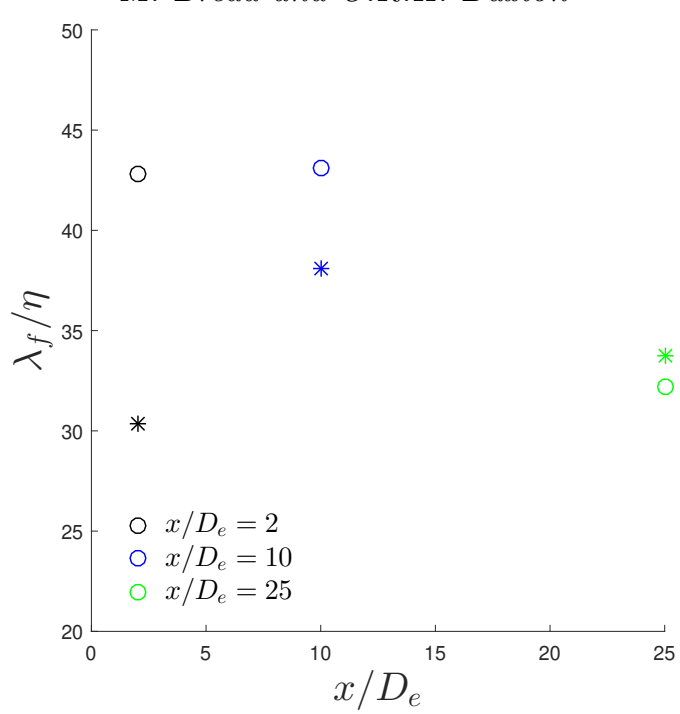

Figure 17: Evolution of the ratio between bulk $\lambda_{f}$ and $\eta$ for round (o) and fractal jet (*).

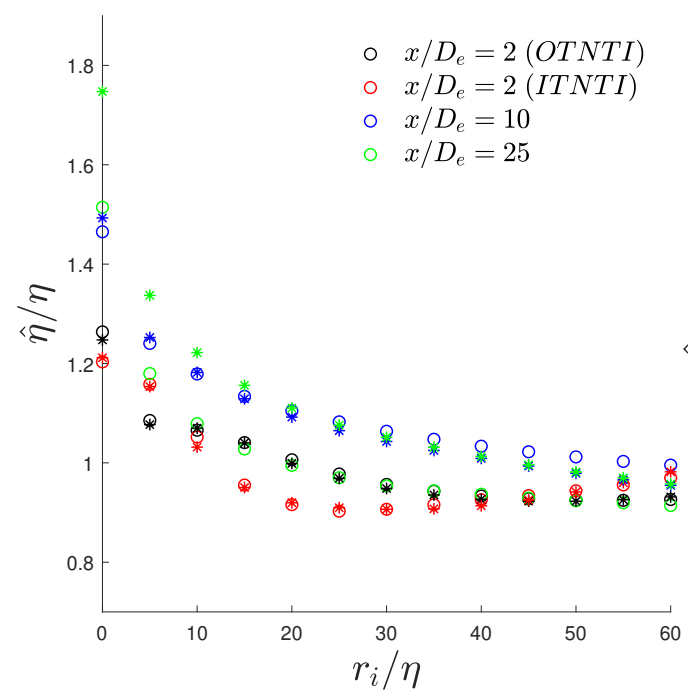

(a)

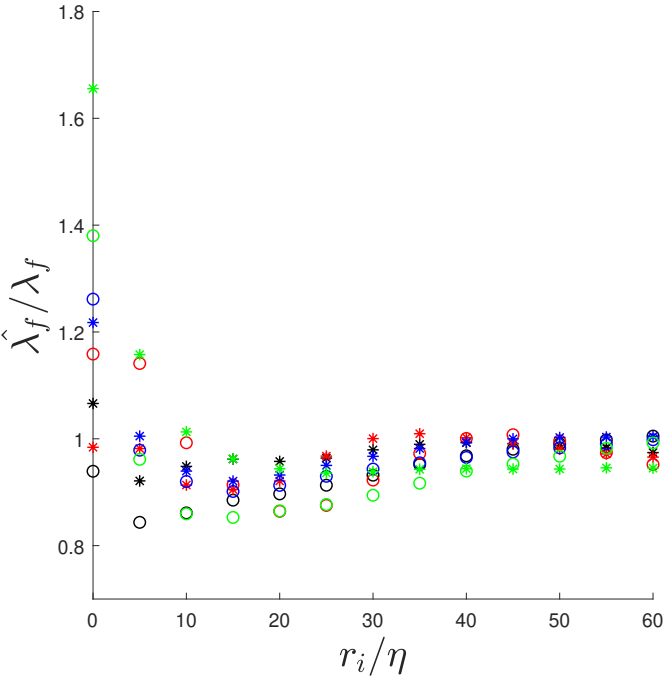

(b)

Figure 18: Evolution of (a) local Kolmogorov and of (b) local Taylor lengthscale for round (o) and fractal jet $(*)$.

to a distance of $\lambda_{f}$ away from the interface, after which it reaches a plateau. This would therefore suggest that the lengthscales evolve inside the TNTI of thickness $\lambda_{f}$, however they do not vary any more once the turbulent bulk is reached. The local $\hat{\eta}$ appears to take slightly longer to reach a plateau compared to $\hat{\lambda}_{f}$, even though the most rapid changes take place within a distance of $\lambda_{f}$ again.

Examining the relationship between the local length scales, the ratio between these two scales changes based on the streamwise location and in general it increases from the irrotational boundary moving towards the turbulent bulk as shown in fig. 19a. At $r_{i} \approx$ 


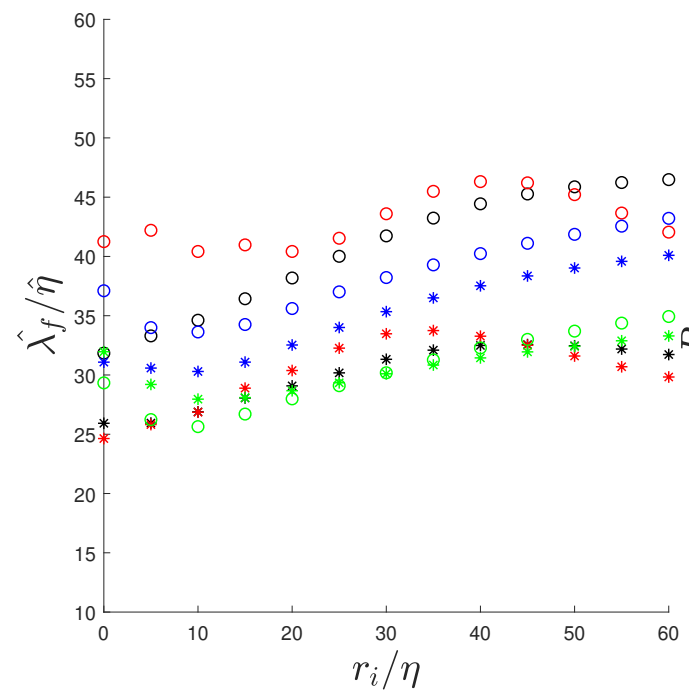

(a)

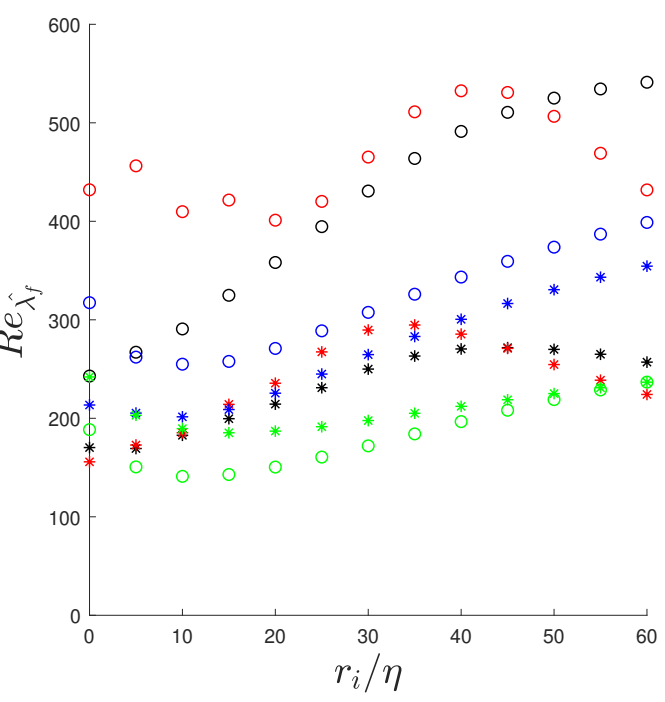

(b)

Figure 19: Evolution of (a) $\hat{\lambda}_{f} / \hat{\eta}$ and of (b) $R e_{\hat{\lambda}_{f}}$ for round (o) and fractal jet (*). The legend is the same as fig. 18

$30-40 \eta$, this ratio decreases for the ITNTI and the OTNTI at $x / D_{e}=2$, however this could be due to the fact that the points furthest away from one interface are approaching the other one. This is also reflected in fig. 19b, where the evolution of the local Taylor Reynolds number is examined. This is calculated as $R e_{\hat{\lambda}_{f}}=\hat{u_{x}^{\prime}} \hat{\lambda_{f}} / \nu$, where $\hat{u_{x}^{\prime}}$ is the root-mean-square of the streamwise velocity fluctuations calculated in the same region as $\hat{\lambda}_{f}$. As shown, $R e_{\hat{\lambda}_{f}}$ increases with increasing distance from the irrotational boundary, reflecting the behaviour of $\hat{\lambda}_{f} / \hat{\eta}$. This shows that there is a significant scale evolution from the TNTI moving towards the turbulent bulk and that choosing the mean bulk value to describe the state of a turbulent flow may not be representative.

Now a potential reason for such evolution is examined. The TNTI separates the nonturbulent from the turbulent fluid, acting like a "barrier" between the two types of flow, as mentioned in section 4.1.3. This "blocking" effect by the TNTI is similar to the concept of "shear sheltering", discussed at length in Hunt \& Durbin (1999), with the contrast that vortical motions are only present on one (the turbulent) side of the interface. The "blocking effect" of the TNTI may be hypothesised to have a similar role to a wall in a turbulent boundary layer, which would lead to an increase of the small-scale anisotropy of the flow near the TNTI. Hence, this would affect the velocity gradients and as a consequence $\varepsilon, \lambda_{f}$ and $\eta$, since the interface-normal direction, similar to the wall-normal direction of a turbulent boundary layer, is highly anisotropic. In addition, the thin layer closest to the wall of a wall-bounded flow is dominated by viscous processes as is the outermost part of the TNTI. Bechlars \& Sandberg (2017) showed that, for a compressible boundary layer, the wall-normal velocity gradient (and hence its anisotropy) would be dominant at the wall with respect to the other terms of the velocity gradient tensor and it would decrease moving away from the viscous sublayer. We thus examine the state of 


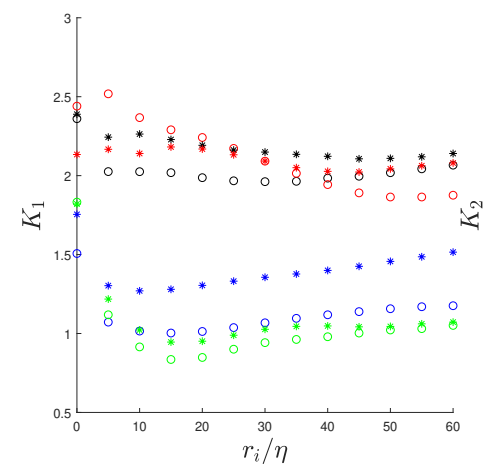

(a)

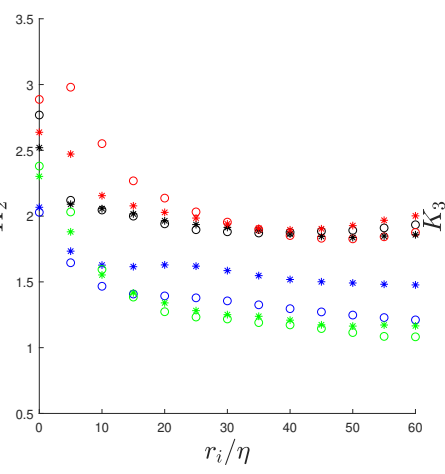

(b)

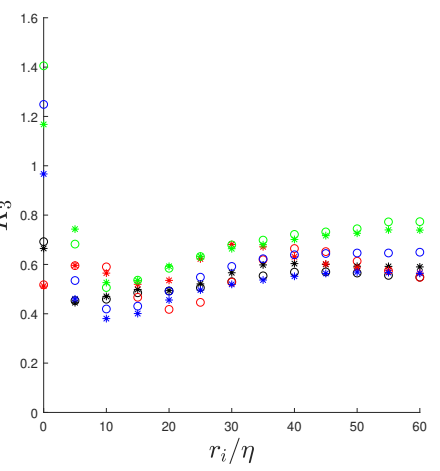

(c)

Figure 20: Evolution of (a) $K_{1}$, (b) $K_{2}$ and (c) $K_{3}$ for round (o) and fractal jet (*). The legend is the same as fig. 18

the anisotropy in the TNTI through three ratios $K_{i}$

$$
K_{1}=2 \frac{\overline{\left(\partial u_{x} / \partial x\right)^{2}}}{\overline{\left(\partial u_{r} / \partial x\right)^{2}}}, K_{2}=2 \frac{\overline{\left(\partial u_{x} / \partial x\right)^{2}}}{\overline{\left(\partial u_{\theta} / \partial x\right)^{2}}}, K_{3}=2 \frac{\overline{\left(\partial u_{x} / \partial x\right)^{2}}}{\overline{\left(\partial u_{x} / \partial r\right)^{2}}},
$$

to verify how the velocity gradients evolve from the irrotational boundary towards the turbulent bulk.

As shown in fig. 20, the anisotropy is the greatest at the irrotational boundary for all streamwise locations and for all the three $K_{i}$. The trends of $K_{1}$ and $K_{2}$ appear to decrease moving further downstream, as expected due to the reduced influence of the coherent structures, however an isotropic state where $K_{i}=1$ is not yet reached for all ratios. Moreover, at $x / D_{e}=2$ the flow is constrained by the presence of two TNTIs, which may not leave enough space to the flow to recover any "bulk" isotropy. In general a plateau or a constant trend is found after a distance of $\lambda_{f}$. It appears that these anisotropy ratios vary mainly inside the TNTI as $\hat{\lambda}_{f}$ and $\hat{\eta}$. Therefore, this suggests that the local quantities are strongly influenced by the anisotropy of the flow especially inside the TNTI.

\section{Discussion and conclusions}

It was found that the break-up of the coherent structures by the fractal orifice affects the tortuosity of the ITNTI and also reduces the thickness of the OTNTI in the near field. The excursions of the TNTI are similar for both jets when the interface moves towards the turbulent side whilst they vary if the interface moves towards the nonturbulent side. Whilst the excursions of the OTNTI were comparable between the two jets it was shown that the round jet, with a stronger contribution from the coherent structures, sees more common large-scale excursions of the ITNTI into the potential core. This leads to a slightly more tortuous ITNTI for the round jet, with a presumably correspondingly greater surface area over which entrainment via nibbling may take place. It thus seems that the difference in entrainment rates in the near fields of both jets reported in Breda \& Buxton (2018b) may be a result of both enhanced engulfment (largescale excursions of the TNTI are likely to promote this) and enhanced nibbling through a more contorted interface. Using a box-counting technique, it was verified that the TNTI follows a scaling of $N \sim B_{s}^{-6 / 5}$ in the regions studied, whilst only the fractal jet appears 
to start approaching the Kolmogorov scaling of $N \sim B_{s}^{-4 / 3}$ in the furthest downstream measurement station. Despite $N \sim B_{s}^{-6 / 5}$ having been linked before to the presence of non-equilibrium dissipation, the fractal jet was previously found to have both this type of dissipation (Breda \& Buxton 2018b) and a Kolmogorov scaling for the fractal dimension of the TNTI. This perhaps suggests that there is a difference between examining the fractal dimension of the TNTI in a streamwise - radial plane as opposed to an azimuthal - radial plane.

We report here the spatial development of the $Q-R$ j.p.d.f. for an axisymmetric turbulent jet for the first time. Its spatial development, like that for grid-generated turbulence in Gomes-Fernandes et al. (2014), contrasts to the tear-drop shaped distribution being observed throughout the near-wake region of a statistically two-dimensional bluff body wake in Buxton et al. (2017). An analysis of the microstructure of the jets indicated that, in the near field, turbulence is constrained between the ITNTI and of the OTNTI. This inhibits the background non-local strain field, which occurs concurrently to a suppression of the inviscid vortex stretching term. Comparing the present work with that of Gomes-Fernandes et al. (2014), it is confirmed that the $Q-R$ j.p.d.f. develops with streamwise distance from the jet exit. Other similarities are represented by the initial balance between the inviscid production and destruction of strain-rate and enstrophy. However, some differences are present in the near field, where for a round jet there are two TNTIs and the flow is initially not fully turbulent. In fact, the symmetrical j.p.d.f. of $Q-R$ at $x / D_{e}=2$ was not found by Gomes-Fernandes et al. (2014).

By comparing the round and fractal jets a comparison was also made between two flows with identical global Reynolds numbers but different coherent structures embedded into the flow, and hence initial conditions. The ratio $\lambda_{f} / \eta$ is indicative of the range of scales present within the flow, and hence the local turbulent Reynolds number. Despite the different initial conditions, and a different range of length scales present i.e. different $\lambda_{f} / \eta$ ratios, the velocity-gradient phenomenology of the round and fractal jets was extremely similar at the intermediate measurement station $x / D_{e}=10$. This highlights the short "memory" that the microscales of the flow have of their initial conditions and the robustness of the $Q-R$ statistical distribution.

The analysis then moved towards understanding how the velocity gradients varied through the TNTI of nominally axisymmetric jets, into the turbulent bulk. It was found that in most cases the contours of the joint p.d.f.s between the invariants $Q$ and $R$ start collapsing after a distance of $\lambda_{f}$ away from the irrotational boundary, suggesting that the microscale structure of the jet evolves across the interface before becoming comparable to the turbulent bulk. This variation of the behaviour of the velocity-gradient invariants in the interface region is coupled to a sharp increase in the small-scale anisotropy. This behaviour of the velocity gradients results in a substantial change of the local Taylor and Kolmogorov lengthscales, $\hat{\lambda}_{f}$ and $\hat{\eta}$ respectively, with distance from the irrotational boundary. It appears that a distance of $\lambda_{f}$ is necessary for the lengthscales to have a comparable value spatially. The evolution of turbulent properties also takes place across a distance of $\lambda_{f}$ for both jets, indicating that the initial conditions may only impact large-scale phenomena directly, whilst the fine structure of the flow remains relatively unaltered. This does not, however, discount an indirect effect on small-scale processes such as nibbling through an increase in the surface area of the TNTI over which it may take place. 
The authors gratefully acknowledge EPSRC for funding through EPSRC Grant No. $\mathrm{EP} / \mathrm{L} 023520 / 1$.

\section{REFERENCES}

Attili, A., Cristancho, J. C. \& Bisetti, F. 2014 Statistics of the turbulent/non-turbulent interface in a spatially developing mixing layer. Journal of Turbulence 15 (9), 555-568.

Bechlars, P. \& SANDBERG, R. D. 2017 Variation of enstrophy production and strain rotation relation in a turbulent boundary layer. Journal of Fluid Mechanics 812, 321-348.

Betchov, R. 1956 An inequality concerning the production of vorticity in isotropic turbulence. Journal of Fluid Mechanics pp. 497-504.

Bisset, D. K., Hunt, J. C. R. \& Rogers, M. M. 2002 The turbulent/non-turbulent interface bounding a far wake. Journal of Fluid Mechanics 451, 383-410.

Blackburn, H. M., Mansour, N. N. \& Cantwell, B. J. 1996 Topology of fine-scale motions in turbulent channel flow. Journal of Fluid Mechanics 310, 269-292.

Breda, M. \& Buxton, O. R. H. 2018a Effects of multiscale geometry on the large-scale coherent structures of an axisymmetric turbulent jet. Journal of Visualization 21 (4), 525532 .

Breda, M. \& Buxton, O. R. H. $2018 b$ Influence of coherent structures on the evolution of an axisymmetric turbulent jet. Physics of Fluids 30 (035109), 1-24.

Buxton, O. R. H. 2015 Modulation of the velocity gradient tensor by concurrent large-scale velocity fluctuations in a turbulent mixing layer. Journal of Fluid Mechanics 777, 1-12.

Buxton, O. R. H., Breda, M. \& Chen, X. 2017 Invariants of the velocity-gradient tensor in a spatially developing inhomogeneous turbulent flow. Journal of Fluid Mechanics 817, $1-20$.

Buxton, O. R. H., Breda, M. \& Dhall, K. 2019 Importance of small-scale anisotropy in the turbulent/nonturbulent interface region of turbulent free shear flows. Physical Review Fluids 4 (3), 34603.

Buxton, O. R. H. \& Ganapathisubramani, B. 2010 Amplification of enstrophy in the far field of an axisymmetric turbulent jet. Journal of Fluid Mechanics 651, 483-502.

Buxton, O. R. H., Laizet, S. \& Ganapathisubramani, B. 2011 The effects of resolution and noise on kinematic features of fine-scale turbulence. Experiments in Fluids 51, 1417-1437.

Chacin, J. M. \& Cantwell, B. J. 2000 Dynamics of a low Reynolds number turbulent boundary layer. Journal of Fluid Mechanics 404, 87-115.

Cheng, W. 1996 Study of the velocity gradient tensor in turbulent flows. PhD thesis, Stanford University.

Chertkov, M., Pumir, A. \& Shraiman, B. I. 1999 Lagrangian tetrad dynamics and the phenomenology of turbulence. Physics of Fluids 11 (8), 2394-2410.

Chong, M. S., Perry, A. E. \& Cantwell, B. J. 1990 A general classification of three-dimensional flow fields. Physics of Fluids A: Fluid Dynamics 2 (5), 765-777.

Corrsin, S. \& Kistler, A. L. 1954 Free-stream boundaries of turbulent flows. Tech. Rep.. NACA.

Dahm, W. J. A. \& Dimotakis, P. E. 1987 Measurements of entrainment and mixing in turbulent jets. AIAA Journal $\mathbf{2 5}$ (9), 1216-1223.

De Silva, C. M., Philip, J. \& Marusic, I. 2013 Minimization of divergence error in volumetric velocity measurements and implications for turbulence statistics. Experiments in Fluids $54(7), 1-17$.

Dimotakis, P. E. 2000 The mixing transition in turbulent flows. Journal of Fluid Mechanics 409, 69-98.

Discetti, S. \& Astarita, T. 2012 Fast 3D PIV with direct sparse cross-correlations. Experiments in Fluids 53, 1437-1451.

Elsinga, G. E., Ishihara, T., Goudar, M. V., Da Silva, C. B. \& Hunt, J. C. R. 2017 The scaling of straining motions in homogeneous isotropic turbulence. Journal of Fluid Mechanics 829, 31-64.

Elsinga, G. E. \& Marusic, I. 2010 Universal aspects of small-scale motions in turbulence. Journal of Fluid Mechanics 662, 514-539. 
Fiscaletti, D., Attili, A., Bisetti, F. \& Elsinga, G. E. $2016 a$ Scale interactions in a mixing layer - the role of the large-scale gradients. Journal of Fluid Mechanics 791, 154-173.

Fiscaletti, D., Elsinga, G. E., Attili, A., Bisetti, F. \& Buxton, O. R. H. $2016 b$ Scale dependence of the alignment between strain rate and rotation in turbulent shear flow. Physical Review Fluids 1 (064405), 1-14.

Gomes-Fernandes, R., Ganapathisubramani, B. \& Vassilicos, J. C. 2014 Evolution of the velocity-gradient tensor in a spatially developing turbulent flow. Journal of Fluid Mechanics 756, 252-292.

Goto, S. \& VAssilicos, J. C. 2016 Local equilibrium hypothesis and Taylor's dissipation law. Fluid Dynamics Research 48 (021402), 1-17.

Hamlington, P. E., Schumacher, J. \& Dahm, W. J. A. 2008 Direct assessment of vorticity alignment with local and nonlocal strain rates in turbulent flows. Physics of Fluids 20 (111703), 1-5.

Herman, G. T. \& Lent, A. 1976 Iterative Reconstruction Algorithms. Computer Biological Medision 6, 273-294.

Ho, C. \& Gutmark, E. 1987 Vortex induction and mass entrainment in a small-aspect-ratio elliptic jet. Journal of Fluid Mechanics 179, 383-405.

Holzner, M. \& Lüthi, B. 2011 Laminar Superlayer at the Turbulence Boundary. Physical Review Letters 106 (134503), 1-4.

Hunt, J. \& Durbin, P. 1999 Perturbed vortical layers and shear sheltering. Fluid Dynamics Research 24, 375-404.

Ishihara, T., Kaneda, Y. \& Hunt, J. C. R. 2013 Thin Shear Layers in High Reynolds Number Turbulence-DNS Results. Flow, Turbulence and Combustion 91, 895-929.

LANE-SerfF, G. 1993 Investigation of the fractal structure of jets and plumes. Journal of Fluid Mechanics 249, 521-534.

Mandelbrot, B. B. 1982 The fractal geometry of nature. W. H. Freeman and Company.

Mistry, D., Dawson, J. R. \& Kerstein, A. R. 2018 The multi-scale geometry of the near field in an axisymmetric jet. Journal of Fluid Mechanics 838, 501-515.

Mistry, D., Philip, J., Dawson, J. R. \& Marusic, I. 2016 Entrainment at multi-scales across the turbulent/non-turbulent interface in an axisymmetric jet. Journal of Fluid Mechanics 802, 690-725.

Mungal, M. G., Karasso, P. S. \& Lonzano, A. 1991 The Visible Structure of Turbulent Jet Diffusion Flames: Large-Scale Organization and Flame Tip Oscillation. Combustion Science and Technology 76, 165-185.

Nedić, J., Vassilicos, J. C. \& Ganapathisubramani, B. 2013 Axisymmetric turbulent wakes with new nonequilibrium similarity scalings. Physical Review Letters 111 (144503), 1-5.

Novara, M., Batenburg, K. J. \& Scarano, F. 2010 Motion tracking-enhanced MART for tomographic PIV. Measurement Science and Technology 21 (035401), 1-18.

Paul, I., Papadakis, G. \& Vassilicos, J. C. 2017 Genesis and evolution of velocity gradients in near-field spatially developing turbulence. Journal of Fluid Mechanics 815, 295-332.

van Reeuwijk, M. \& Holzner, M. 2014 The turbulence boundary of a temporal jet. Journal of Fluid Mechanics 739, 254-275.

DA Silva, C. B. 2009 The behavior of subgrid-scale models near the turbulent/nonturbulent interface in jets. Physics of Fluids 21 (8), 081702:1-4.

DA Silva, C. B. 2010 Coherent structures and their signature on the Q-R invariant map near a turbulence / non-turbulent interface.

da Silva, C. B., Hunt, J. C. R., Eames, I. \& Westerweel, J. 2014 Interfacial Layers Between Regions of Different Turbulence Intensity. Annual Review of Fluid Mechanics 46 (1), 567-590.

DA Silva, C. B. \& Pereira, J. C. F. 2008 Invariants of the velocity-gradient, rate-of-strain, and rate-of-rotation tensors across the turbulent/nonturbulent interface in jets. Physics of Fluids 20 (055101), 1-18.

DA Silva, C. B. \& Taveira, R. R. 2010 The thickness of the turbulent/nonturbulent interface is equal to the radius of the large vorticity structures near the edge of the shear layer. Physics of Fluids 22 (121702), 1-4.

Silva, T. S., Zecchetto, M. \& DA Silva, C. B. 2018 The scaling of the turbulent/nonturbulent interface at high Reynolds numbers. Journal of Fluid Mechanics 843, 156-179. 
Soria, J., Sondergandd, R., Cantwell, B. J., Chong, M. S. \& Perry, A. E. 1994 A study of the fine-scale motions of incompressible time-developing mixing layers. Physics of Fluids 6 (2), 871-884.

Sreenivasan, K. R. \& Meneveau, C. 1986 The fractal facets of turbulence. Journal of Fluid Mechanics 173, 357-386.

Sreenivasan, K. R., Ramshankar, R. \& Meneveau, C. 1989 Mixing, entrainment and fractal dimensions of surfaces in turbulent flows. Proceedings of the Royal Society A: 421, 79-108.

TAYlor, G. I. 1938 The spectrum of turbulence. In Proceedings of the Royal Society of London., pp. 476-490. The Royal Society.

Tsinober, A. 2009 An Informal Conceptual Introduction to Turbulence, 2nd edn. Springer.

Tsinober, A., Kit, E. \& Dracos, T. 1992 Experimental Investigation of the Field of VelocityGradients in Turbulent Flows. Journal Of Fluid Mechanics 242, 169-192.

Vassilicos, J. C. 2015 Dissipation in Turbulent Flows. Annual Review of Fluid Mechanics 47, $95-114$.

Vieillefosse, P. 1982 Local interaction between vorticity and shear in a perfect incompressible fluid. Journal de Physique $\mathbf{4 3}$ (6), 837-842.

Watanabe, T., Jaulino, R., Taveira, R. R., Da Silva, C. B., Nagata, K. \& Sakai, Y. 2017 Role of an isolated eddy near the turbulent/non-turbulent interface layer. Physical Review Fluids 2 (9), 94607.

Watanabe, T., Sakai, Y., Nagata, K., Ito, Y. \& Hayase, T. 2015 Turbulent mixing of passive scalar near turbulent and non-turbulent interface in mixing layers. Physics of Fluids 27 (085109).

Westerweel, J., Funushima, C., Pedersen, J. M. \& Hunt, J. C. R. 2005 Mechanics of the Turbulent-Nonturbulent Interface of a Jet. Physical Review Letters 95 (174501), 1-4.

Westerweel, J., Fukushima, C., Pedersen, J. M. \& Hunt, J. C. R. 2009 Momentum and scalar transport at the turbulent/non-turbulent interface of a jet. Journal of Fluid Mechanics 631, 199-230.

WiEneKe, B. 2008 Volume self-calibration for 3D particle image velocimetry. Experiments in Fluids 45, 549-556.

Worth, N. A., Nickels, T. B. \& Swaminathan, N. 2010 A tomographic PIV resolution study based on homogeneous isotropic turbulence DNS data. Experiments in Fluids 49, 637-656.

Zноu, Y. \& VAssiLicos, J. C. 2017 Related self-similar statistics of the turbulent/non-turbulent interface and the turbulence dissipation. Journal of Fluid Mechanics 821, 440-457. 